\title{
TEORIE DELLA GIUSTIZIA COSTITUZIONALE E LEGITTIMAZIONE DEGLI ORGANI DI GIUSTIZIA COSTITUZIONALE ${ }^{1}$
}

LuCA MEZzETTI

Professore ordinario di Diritto costituzionale nella Facoltà di Giurisprudenza dell’Università di Bologna (Italia) luca.mezzetti@unibo.it

La problematica relativa alla legittimazione democratica della giurisdizione costituzionale appare di fondamentale rilevanza in uno Stato democratico. La riconducibilità al popolo della giurisdizione costituzionale in quanto manifestazione ed esercizio della sovranità statale si rivela imprescindibile soprattutto ove alla giurisdizione medesima sia conferita l'attribuzione di annullare in quanto costituzionalmente illegittime le leggi approvate dalla rappresentanza popolare eletta dal corpo elettorale. L'elaborazione teorica finalizzata a concretarsi nella sussunzione della giustizia costituzionale entro lo schema della legittimazione democratica fondata sulla sovranità popolare ha come obiettivo di impedire che la giurisdizione costituzionale, istituita a salvaguardia della costituzione, diventi uno strapotere incontrollato rispetto agli altri e in tal modo minacci la struttura costituzionale democratica, invece di tutelarla. Essa deve riuscire a far sì che il controllo che esercita su altri poteri legittimati dal popolo, specialmente su quello del legislatore, possa essere considerato a sua volta come proveniente dal popolo. E deve anche realizzare le condizioni che non impediscano ma rendano positivamente possibile alla giurisdizione costituzionale, custode della costituzione approvata e sostenuta dal popolo, di agire con efficacia [E. W. Böckenförde, 2006, 652].

La teoria della giustizia costituzionale si configura in tal modo quale anello di congiunzione essenziale tra la teoria della Costituzione da una parte e la teoria dell'interpretazione costituzionale dall'altra. Il continuum teoria della Costituzioneteoria della giustizia costituzionale-teoria dell'interpretazione costituzionale denota la stretta interdipendenza dei segmenti che lo compongono e l'osmosi reciproca dei fattori che lo caratterizzano, ed implica necessariamente un approccio sistemico

\footnotetext{
${ }^{1}$ Trabajo preparado por el profesor Luca Mezzetti en 2009 para el X Congreso Iberoamericano de Derecho Constitucional celebrado en Lima, Perú, y enviado para ser publicado como Documento en Estudios Constitucionales, lo que realizamos con gusto, por las interesantes y valiosas reflexiones realizadas en el mismo.
} 
capace di tradursi nell'inquadramento del secondo elemento del trinomio alla luce del primo (la teoria della giustizia costituzionale nell'ottica della teoria della Costituzione) e, come vedremo nel paragrafo successivo, del terzo elemento del trinomio alla luce del secondo (la teoria dell'interpretazione costituzionale alla luce della teoria della giustizia costituzionale).

Tale approccio è stato variamente concepito ad opera delle diverse posizioni dottrinali che hanno affrontato la problematica in precedenza delineata.

\section{Nella DOTTRINA ITALiANA}

Secondo la teoria realista elaborata da V. Crisafulli, nessun modello di giustizia costituzionale può vantare piena legittimità democratica, e ciò vale anche per il sistema della giurisdizione costituzionale italiana:

la Corte non è organo rappresentativo, anche se per un terzo di formazione elettiva e per un altro terzo composta da giudici nominati da un organo elettivo, quale il Capo dello Stato (a sua volta però non immediatamente rappresentativo del popolo, ma -che è cosa diversa-rappresentante "lunità nazionale"). Vero è peraltro che se anche il sindacato di costituzionalità fosse diffuso, spettando ad ogni giudice, non per questo l'antinomia sarebbe evitata. Ma d'altronde, più in generale, non è forse l'intero sistema della giurisdizione estraneo al principio democratico? Non è forse quello dei giudici (di qualsiasi gindice) un potere privo di legittimazione democratica, ed irresponsabile? [V. Crisafulli, 1978, 73].

L'(apparente) antinomia si supera solo, tuttavia, secondo l'Autore, considerando l'accennata contraddizione inevitabile ed immanente alla Costituzione liberal-democratica:

in realtà, se osserviamo le cose serenamente, la giustizia costituzionale non tanto rappresenta un'antinomia, quanto piuttosto un'alterazione dello schema democratico-parlamentare, ma un'alterazione intenzionalmente posta in essere dai costituenti, in funzione di un preciso disegno politico, nella quale si esprime al più alto livello quella componente liberale, $e$ quindi garantista, che qualifica il regime democratico costituzionalmente adottato.

Bisogna riconoscere, infatti, che la mancanza di legittimazione democratica che si riscontra nella struttura della nostra Corte costituzionale (come di solito degli organi similari esistenti in altri ordinamenti) è inevitabile ed anzi in qualche misura necessaria per assicurare l'effettiva estraneità del "controllore" rispetto al "controllato". Se le assemblee legislative e la Corte costituzionale derivassero egualmente dall'investitura popolare, e tanto più se questultima fosse diretta ed esclusiva emanazione di quelle, si realizzerebbe, bensì, un alto grado di omogeneità politica e quasi un continuum tra $i$ due poteri, ma a prezzo di un affievolimento del controllo, che finirebbe per rassomigliare ad un autocontrollo $[V$. Crisafulli, 1978, 73-74].

La Corte costituzionale, anzi, è fattore che converge ai fini della caratterizzazione e definizione della nostra forma di governo: 
Corte costituzionale, referendum, poteri rimessi alla libera iniziativa e determinazione del Presidente della Repubblica, decentramento legislativo regionale, sono altrettanti elementi che si illuminano a vicenda, concorrendo complessivamente a specificare il proprium della nostra forma di governo: che si fonda sulla souranità popolare, e non delle assemblee; dalla quale esula il principio della onnipotenza della legge; che sottrae al Parlamento il monopolio della legislazione; che prevede una serie di limiti e di contrappesi al potere politico dello stesso Parlamento [V. Crisafulli, 1978, 74].

Tale caratterizzazione si manifesta, secondo una visione organicistica e antivolontaristica della Costituzione e dei rapporti fra giustizia costituzionale e assemblee rappresentative [F. Modugno, 1982, 19 ss.], mediante un'interpretazione della Costituzione privilegiata rispetto a quella del potere legislativo, attraverso la funzione esplicata dall'organo di garanzia come di primo indirizzo e guida rispetto al legislatore: il ricorso da parte della Corte costituzionale a modalità di interpretazione sistematica fondate essenzialmente sulla tecnica della ponderazione e bilanciamento dei principi e valori costituzionali consente di avvertire che,

attraverso un'opera apparentemente ermeneutica, l'organo di garanzia tenderebbe a trasformarsi, come dice qualcuno, in potere costituente in seduta permanente (Antonio Baldassarre). Il custode della Costituzione si trasfigura, in realtà, come pure è stato felicemente detto, in moderatore di conflitti sociali (Leopoldo Elia, Carlo Mezzanotte) [F. Modugno, 1982, 52-53, grassetti nostri].

La dilatazione e creazione degli strumenti operativi, non soltanto decisori, ed il loro uso discrezionale ad opera dell'organo di giustizia costituzionale costituirebbero secondo tale posizione dottrinale indici rivelatori della natura essenzialmente politica del controllo di costituzionalità: la collateralità della funzione della Corte costituzionale rispetto alla legislazione parlamentare "non toglie che essa abbia e non possa non manifestare una sua valenza politico-legislativa" [F. Modugno, 1982, 54], che tende a tradursi in una funzione di impulso e di iniziativa (in senso atecnico) qualificata, che ha contribuito a costruire e ad accentuare "il suo ruolo politico di mediatrice degli interessi sostanziali e di moderatrice dei conflitti" [F. Modugno, 1982, 99, grassetti nostri]. Alla funzione di svolgimento costituzionale sarebbero chiamati unitariamente, secondo tale prospettiva, sia la Corte costituzionale che il legislatore: si tratta di argomentazione non esente da osservazioni critiche formulate da diverso angolo visuale [G. Zagrebelsky, 1982, 103 ss. e 1988, 39 ss.], secondo cui

La Costituzione sarebbe insomma non un vincolo esterno, che pone limiti insuperabili, ma si manifesta indifferente rispetto a ciò che si svolge all'interno, bensì una forza interna, animatrice di tutta quanta l'attività legislativa (...) Il ruolo vero della Corte sarebbe dunque di partecipare al processo rivolto a rendere effettiva tale forza interna, in un 
processo che, come è chiaro, non ha nulla di giurisdizionale, se non ci si fa ingannare dalle nude ed illusorie apparenze, ma ha tutto di politico, nel senso almeno in cui è politica l'attuazione della Costituzione [G. Zagrebelsky, 1982, 150].

La posizione dottrinale da ultimo richiamata, riconducibile al novero della visione contrattualistica-volontaristica degli accennati rapporti, distingue, quanto al coinvolgimento della Corte costituzionale nel processo legislativo ed al ruolo normativo della medesima, tra la produzione immediata di regole di diritto non legislativo (Corte-legislatore) attraverso sentenze-legge e la partecipazione al processo legislativo (Corte-co-legisaltore) attraverso sentenze-indirizzo al legislatore. In considerazione del fatto che

il controllo di costituzionalità, con tutto ciò che presuppone e comporta, è un limite, quanto al principio su cui si fonda, e quanto al contenuto della sua azione, al principio maggioritario. In una costituzione non monistica, che accoglie in sé diversi principi ispiratori, l'organo investito della funzione di controllo non partecipa conseguentemente di un fondamento analogo a quello degli organi politicamente attivi ove si esprime il principio di maggioranza [G. Zagrebelsky, 1982, 111],

$\mathrm{Al}$ quesito relativo alla esistenza di ragioni istituzionali positive che giustifichino una compressione della sfera discrezionale del legislatore da parte dell'organo di giustizia costituzionale, l'Autore fornisce una risposta negativa, con la sola eccezione degli indirizzi al legislatore consistenti nell'affermazione ed esplicitazione di obblighi direttamente derivanti dalla Costituzione [G. Zagrebelsky, 1982, 142]. Adottando modello di riferimento opposto rispetto a quello precedentemente menzionato, si sottolinea

La distinzione netta tra il ruolo degli organi di produzione legislativa e il ruolo del giudice di costituzionalità delle leggi, con il conseguente apprezzamento delle diversità di organizzazione, nei due casi, come espressione di unirriducibile diversità di funzioni: l'una, quella legislativa, libera sul piano giuridico-costituzionale, fino al punto in cui non incontra il limite della Costituzione; l'altra, vincolata e posta là dove sta il limite di compatibilità con la Costituzione di tutte le possibili politiche che l'attuale quadro costituzionale è idoneo a recepire [G. Zagrebelsky, 1982, 150-151].

Tale visione non entra in rotta di collisione con l'accentuato carattere programmatico della Costituzione italiana e risulta anzi funzionale al recupero almeno parziale, nell'ambito dello Stato sociale di diritto, delle caratteristiche di certezza, stabilità ed obiettività del diritto che l'impatto dello Stato sociale interventista aveva contribuito ad erodere pericolosamente:

A ciò soccorre la creazione di una nuova istanza, legata a valori più stabili, che possa recuperare, su un piano non più solo formale ma ora anche sostanziale, le caratteristiche di obiettività della legge, travolte in misura crescente dalle esigenze dello stato sociale 
interventista. In questottica, si ritiene facilmente giustificabile la scelta a favore di una configurazione giurisdizionale (e non politica) dell'organo di garanzia costituzionale e così pure il collegamento del controllo di costituzionalità delle leggi con l'esercizio della funzione giurisdizionale comune e quindi con la garanzia delle posizioni soggettive che neppure le esigenze di intervento sociale dell'epoca attuale consentono di sacrificare [G. Zagrebelsky, 1982, 154, grassetto nostro].

Attenendosi ai valori costituzionali e rendendoli oggetto di interpretazione animata da coscienza storica senza che ciò implichi concorrenza o competizione con il legislatore,

la Corte può mostrare nei fatti la specificità e l'essenzialità della sua funzione. (...) Una funzione di richiamo a valori d'insieme tanto più importante quanto maggiore è la tendenza della funzione politica in senso stretto a disperdersi in decisioni mediatrici caso per caso, in cui è difficile scorgere l'esistenza di un qualsiasi progetto $[G$. Zagrebelsky, 1982, 155-156, grassetti nostri].

La Corte costituzionale, "propugnatrice di valori e principi d'insieme" [G. Zagrebelsky, 1982, 156], "portatrice fedele dei valori del diritto" [G. Zagrebelsky, 1988, 68], deve cercare la propria legittimazione attraverso la propria opera, promuovendo "nella vita politica l'indispensabilità della propria funzione, essendo partecipe consapevole, a questo scopo e senza coinvolgimenti estranei alla propria posizione imparziale, degli sviluppi politici e del loro significato" [G. Zagrebelsky, 1988, 68].

La problematica della legittimazione democratica dei tribunali costituzionali, "vere e proprie longae manus nel tempo del potere costituente", è stata altresì affrontata da A. Ruggeri e A. Spadaro [A. Ruggeri-A. Spadaro, 2004, 10 ss.] che, rovesciando l'impostazione tradizionale ed interrogandosi in primo luogo sulla legittimazione in sé del regime democratico, forniscono una risposta articolata in sette punti.

Una vera democrazia, ad avviso degli Autori,

non trova solo in sé medesima la fonte della sua legittimazione, avendo invece necessariamente bisogno di un "sistema di valori metademocratici" di riferimento a cui soggiacere essa stessa. Tale sistema in gran parte è, appunto, la Costituzione. Si accenna, così, al principio della c.d. "doppia legittimazione" che sta all'origine della Costituzione democratica o della democrazia costituzionale, l'unico che -in qualche modo-può "spiegare"le funzioni dei tribunali costituzionali e l'anomalia di una loro contrapposizione alle scelte democratico-parlamentari [A. Ruggeri-A. Spadaro, 2004, 11].

La "naturale carenza di legittimazione democratica" delle corti costituzionali deriva dalla loro funzione di custodi della cornice metademocratica di valori che limitano la forza dello stesso regime democratico: garantendo l'equilibrio fra i due 
poli della sovranità popolare da un lato e della supremazia di un sistema di valori costituzionalizzati dall'altro, le corti -pur non esercitando attività di indirizzo politico in senso stretto, si configurano comunque quali "organi giudiziari dotati di forza politica, con funzioni giuridico-arbitrali di custodia-garanzia anche- se non soprattutto - nei confronti degli stessi atti (leggi) in cui si traduce la sovranità popolare" [A. Ruggeri-A. Spadaro, 2004, 11]. Sono tali funzioni garantiste e di custodia che portano le corti costituzionali -pu non elettive- ad operare in nome e nell'interesse di tutti i consociati.

Oltre che arbitro, l'organo di giustizia costituzionale è componente di un sistema equilibrato di pesi e contrappesi caratterizzato dalla presenza di rilevanti funzioni di garanzia conferite ad altri organi (quali il Presidente della repubblica, le autorità amministrative indipendenti ecc.) ed in seno al quale risulta difficile immaginare, secondo Ruggeri-Spadaro, l'ipotesi di una corte che abusi dei propri poteri, tentando di imporre i propri strumenti ermeneutici a fronte dell'interpretazione "colta" dei chierici (giuristi) e "diffusa" dei laici (profani) [A. Ruggeri -A. Spadaro, 2004, 12].

Il quarto fattore che induce gli Autori a relativizzare e circostanziare la carenza di legittimazione democratica degli organi costituzionali viene fatto coincidere con il potere, spettante al titolare del potere di revisione costituzionale, di ripristinare qualunque normativa dichiarata costituzionalmente illegittima dalle corti (o tribunali) costituzionali:

ferma restando l'opportunità di non adottare simili provvedimenti "a ridosso" delle sentenze delle Corti (onde non ingenerare il sospetto di violazione sostanziale del giudicato in fraudem Constitutionis) e riconoscendo sempre l'intangibilità di una parte (nucleo duro irrevisionabile) delle moderne Carte, è bene rammentare che nessuna decisione dei tribunali costituzionali è in grado di resistere ai Parlamenti, nella veste di legislatori costituzionali. Ciò dovrebbe valere, a maggior ragione, nel caso di riproduzione di una legge ingiustamente caducata dalla Corte, quale forma estrema di opposizione ad un annullamento arbitrario [A. Ruggeri-A. Spadaro, 2004, 13].

L'indefettibilità, sotto il profilo logico-giuridico, di "organi di chiusura" in seno al sistema costituzionale e la necessità di riconoscere, "per convenzione", un solo sistema di controlli costituzionali, non essendo configurabile l'ipotesi di organo che possa sindacare la correttezza del percorso interpretativo, da una parte; la natura non autoapplicativa delle decisioni rese dagli organi di giustizia costituzionale e la ordinaria indisponibilità in capo agli organi medesimi di strumenti di attuazione-esecuzione delle proprie pronunce, dall'altra, costituiscono il quinto ed il sesto fattore individuati dagli Autori quali limiti insuperabili alla "sovranità" degli organi di custodia delle Costituzioni. 
Il potere di tali organi, infine, si trova in un rapporto di stretta correlazione e diretta proporzionalità con il consenso sociale sulle Costituzioni e con l'attitudine dei consociati ad osservare i precetti e le chiavi di lettura elaborati in sede giurisprudenziale.

In un'ottica che privilegia la funzione prescrittiva, e non meramente descrittiva, del diritto costituzionale, in cui esso mira

a 'orientare' i consociati verso un predefinito 'sistema di valori' formali (procedure democratiche e separazione fra i poteri) e sostanziali (diritti umani), (...) acquista significato effettivo, e non astratto e irrisorio, la previsione di una giustizia costituzionale" che svolga "una funzione 'stabilizzatrice', 'moderatrice' ed 'equilibratrice' attraverso un intelligente - e dunque ragionevole-aggiornamento nel tempo dei bisogni-valori originari, comunque protetti nel loro nucleo duro intangibile: e questi dovrebbero essere, nello stesso tempo, il fine ed il limite di ogni giurisprudenza costituzionale c.d. evolutiva [A. Ruggeri-A. Spadaro, 2004, 15-17].

\section{Nella DOTTRina TEDESCA}

L'approccio alla problematica della legittimazione democratica della giurisdizione costituzionale ha avuto luogo nella dottrina tedesca sul versante I) costituzionalistico, II) filosofico e III) politologico.

\section{I) L'approccio costituzionalistico}

a) Metodi interpretativi degli organi di giustizia costituzionale

$\beta)$ Delimitazione delle sfere di attribuzioni degli organi di giustizia costituzionale rispetto agli organi rappresentativi

g) Approccio sistemico

\section{a) Metodi interpretativi degli organi di giustizia costituzionale}

Le ragioni addotte a giustificazione della legittimazione democratica della giurisdizione costituzionale attraverso il prisma dei metodi e degli strumenti interpretativi degli organi di giustizia costituzionale vengono trattate $s u b$ par. 6 del presente capitolo. Può anticiparsi in questa sede, a titolo di anticipazione delle osservazioni che si svolgeranno in seguito, che oggetto di analisi è divenuto in particolare, da parte di molteplici posizioni dottrinali e sotto una pluralità di angoli visuali, l'apporto fornito dal Tribunale costituzionale federale al fine della enucleazione ed enunciazione di nuovi diritti e valori in seno al contesto costituzionale della Legge fondamentale, nonché formulando moniti e concrete linee di indirizzo al legislatore e all'Esecutivo in vari settori materiali: tali strumenti portano ad una "colonizzazione della sfera politica" [U. Haltern, 1998, 207], che 
ne viene conseguentemente giuridificata. Tale giuridicizzazione viene assimilata ad una ossificazione in quanto si ritengono estremamente ridotti i margini di correzione-superamento delle decisioni dell'organo di giustizia costituzionale da parte del legislatore: ciò spiega la prevalenza quantitativa degli studi che hanno tentato di individuare la linea di demarcazione delle sfere di attribuzione delle assemblee rappresentative e del Bundesverfassungsgericht, nonché un accettabile criterio giustificativo del potere del primo - organo privo di diretta legittimazione democratica- di annullare atti delle seconde -organi per eccellenza espressione di legittimazione democratica diretta [C. Gusy, 1985; K. Chryssogonos, 1987; C. Landfried, 1984, 1988; J. Jekewitz, 1980; E. Benda, 1979; K. Stern, 1997].

\section{ß) Delimitazione delle sfere di attribuzioni degli organi di giustizia costituzionale rispetto agli organi rappresentativi}

La ubicazione teorica della giurisdizione costituzionale aveva dato luogo ad un vivace dibattito dottrinale ed al sorgere di controversie già all'epoca della Costituzione di Weimar: le argomentazioni addotte non possono infatti riduttivamente riferirsi alla polemica insorta fra Kelsen e Schmitt, alle cui posizioni è già stato dedicato ampio spazio supra (vedi par. 3.8 del presente capitolo). Nel contesto caratterizzato dal campo di tensione instauratosi fra la configurazione del polo della legalità parlamentare e del polo di una legittimità superiore alla stessa Costituzione positiva possono distinguersi la corrente dottrinale positivistica e quella antipositivistica, articolatesi a loro volta in due sub-correnti interne [C. Gusy, 1985; F. Hase, 1980; K.-H. Ladeur, 1980, 103 ss.; K. R. Hinkel, 1984; H. Wendenburg, 1984].

L'antipositivismo conservatore di H. Triepel, R. Smend e di E. Kaufmann si era dimostrato scettico rispetto all'attitudine della Staatsgerichts-barkeit di risolvere conflitti politici a livello centrale; tale scetticismo si era tradotto, come è noto, in una radicale negazione in seno alla posizione elaborata da C. Schmitt. Gli Autori citati, peraltro, mantenevano un atteggiamento possibilista nei confronti del controllo di legittimità costituzionale in via incidentale, esercitato dal Reichsgericht, come si è rilevato in seno al par. 3.6, a partire dal 1921 con le famose sentenze del 28.4.1921, del 21.10.1924 e del 4.11.1925. Il controllo giurisdizionale delle norme trovava al contrario un deciso rigetto da parte della corrente di orientamento socialista dell'antipositivismo, che giungeva a qualificarlo quale "degenerazione del principio dello Stato di diritto" (ne erano qualificati rappresentanti H. Heller e F. Neumann).

Una posizione conciliativa fu assunta dalla scuola positivistica, che nella sua variante liberale (di cui erano eminenti rappresentanti R. Thoma e G. Anschütz) 
e nella sua variante socialista (coincidente in particolare con la posizione di G. Radbruch) si era dimostrata favorevole al conferimento della funzione di controllo ad una corte costituzionale di tipo accentrato.

Più recentemente, l'intento di individuare la linea di demarcazione tra la decisione parlamentare e quella dell'organo di giustizia costituzionale, nonché la ricerca della fonte di legittimazione delle pronunce del secondo, hanno implicato da parte della dottrina tedesca la elaborazione di teorie impostate, rispettivamente, sulla base del principio di separazione dei poteri, della dottrina delle political questions, del principio del judicial self-restraint, del principio della giustapposizione fra diritto e politica, del principio giuridico-funzionale.

Avuto riguardo al primo dei profili indicati, il principio della separazione dei poteri viene configurato -in quanto fondamento del sistema statale delle competenze- quale criterio centrale ai fini della determinazione della posizione della giurisdizione costituzionale nel sistema democratico [L. Adamovich, 1987, 281 ss; W. Knies, 1997, 1155 ss.], giungendosi ad identificare nella giustizia costituzionale una sorta di "quarto potere" di non facile riconducibilità al classico schema della separazione dei poteri [G. Roellecke, 1980, 24 ss.].

Sul secondo dei versanti menzionati, la automatica e meccanica applicabilità al sistema di giustizia costituzionale tedesco della dottrina delle political questions di matrice nordamericana, secondo il quale -come è noto- il controllo di legittimità costituzionale sulle leggi esclude valutazioni di natura politica e un sindacato del giudice costituzionale sull'uso del potere discrezionale da parte del parlamento -è stata valutata dalla dottrina germanica con estrema cautela e scetticismo [K. Schlaich, 1994, 469; K. Stern, 1984, 961 ss.; E. Friesenhahn, 1976; H.-P. Schneider, 1980, 2103 ss.; W. Geiger, 1979, 21; C. Rau, 1996, 228 ss.; G. Leibholz, 1963, 118], rilevandosi da parte di alcune posizioni dottrinali [K. Chryssogonos, 1987, 178; B.-O. Bryde, 1982, 311] come sia in linea di principio estraneo alla Legge fondamentale il concetto di disposizioni costituzionali che non possono fungere da parametro ai fini dell'esercizio del controllo di legittimità costituzionale.

Analogamente, il principio del judicial self-restraint è stato ritenuto di scarsa affidabilità ai fini della identificazione della fonte di legittimazione della giurisdizione costituzionale [R. Dolzer, 1972, 86; K. Chryssogonos, 1987, 174; E.W. Böckenförde, 1991, 192; A. Rinken, 1989, 92; H.-P. Schneider, 1980, 2104; R. Zuck, 1974, 366; D. Murswiek, 1982, 532 ss.; W.-R. Schenke, 1979, 1325; F.-C. Zeitler, 1976, 631].

L'approccio giuridico-funzionale sembra denotare una migliore attitudine sul versante della identificazione degli ambiti propri del legislatore e dell'organo di giustizia costituzionale e dei requisiti di legittimazione di quest'ultimo. È metodo 
analitico seguito, fra gli altri, da E.W. Böckenförde, che identifica la funzione primaria della giurisdizione costituzionale nella protezione della Costituzione:

la giurisdizione costituzionale serve a far salvaguardare la costituzione, nel suo rango e nella sua funzione, da parte di un'istanza indipendente, e a dare validità ai diritti e alle procedure fisse che vi sono garantiti [E. W. Böckenförde, 2006, 631].

Quanto al fondamento dell'idea di una giurisdizione costituzionale nello Stato democratico, osserva il costituzionalista tedesco che

le decisioni di un tribunale costituzionale, intese come strumenti di salvaguardia della costituzione, devono partecipare al cosiddetto primato della costituzione, che produce il nucleo della forza ordinatrice e dell'efficacia giuridica della stessa. Ciò significa al tempo stesso il diritto di prendere l'ultima decisione. Ma (...) un simile diritto abbisogna di una giustificazione in un ordinamento statale che si legittimi sulla base del principio "tutto il potere dello Stato procede dal popolo". Un simile ordinamento non dourebbe affidare al popolo il diritto di ultima decisione? [E. W. Böckenförde, 2006, 631].

Le peculiarità della giurisdizione costituzionale all'interno della struttura dei poteri dello Stato, in particolare rispetto alla giurisdizione normale, vengono individuate dall'Autore nell'oggetto della giurisdizione costituzionale, nelle parti del processo costituzionale, vale a dire dei soggetti che prendono parte alle controversie di diritto costituzionale, nella forza interpretativa del tutto singolare propria della giurisdizione costituzionale.

Quanto al primo profilo, la giurisdizione costituzionale si caratterizza per il fatto di decidere sul contenuto e sull'interpretazione del diritto costituzionale, inteso come diritto politicamente orientato, diritto politico:

se questa giurisdizione percepisce il proprio compito in modo giusto, dal punto di vista funzionale, e corretto, dal punto di vista sostanziale, allora essa ha necessariamente, in questo senso, una dimensione politica. La giurisdizione costituzionale deve allora rendersi conto di questa situazione e affermarsi in essa come giurisprudenza indipendente [E. W. Böckenförde, 2006, 636].

Quanto al secondo profilo, le parti dei conflitti interorganici od intersoggettivi sono rappresentate dai supremi detentori del potere politico, il che significa che

è tipico delle decisioni e delle sentenze di un tribunale costituzionale il non essere coperte dal tetto dell'ordinamento giuridico-legale esistente e il non essere inserite in tale ordinamento, come accade invece nel caso delle controversie tra soggetti che condividono lo stesso diritto o anche in quelle tra Stato e singoli nell'ambito del diritto penale o del diritto amministrativo. Le decisioni di un tribunale costituzionale si rivolgono, in larga misura e almeno da una delle parti, proprio ai titolari dell'ordine giuridico legale, agli attori della politica nel governo e nel parlamento, ai detentori del potere esecutivo e non a coloro che sottostanno a tale potere. Nella maggior parte dei casi tali decisioni implicano 
poi effetti di grande portata, che vanno al di là del caso singolo, tuttavia la riuscita di tali decisioni mediante coazione appare a stento possibile secondo i modi di un'esecuzione regolare. Esse inoltre non possono essere formalmente confermate o soppresse da nessun altro tribunale. La giurisdizione costituzionale, pertanto, ha ben minore sostegno nel sistema giuridico di quanto ne abbia la giurisdizione normale. Essa deve pertanto - agendo anche sul piano del sistema politico- incontrare una sponda istituzionale per il riconoscimento delle sue decisioni, ed è rimandata in larga misura alla sua accettazione [E. W. Böckenförde, 2006, 636-637].

Con riferimento al terzo profilo, la forza interpretativa peculiare della giurisdizione costituzionale

scaturisce dal collegamento di tre elementi: il primato della costituzione, la competenza a dare l'ultima interpretazione vincolante della costituzione stessa, che in ampie parti è indeterminata, infine la mancanza di un canone riconosciuto dei metodi esegetici [E. W. Böckenförde, 2006, 637].

Ciò che realizza la peculiarità della giurisdizione costituzionale, in ultima istanza,

è il suo riferirsi al processo politico, salvaguardandolo e delimitandolo; lo salvaguardia, fornendogli una relativa autonomia rispetto agli ordini sociali e alle diverse condizioni del possesso; lo delimita mediante le garanzie e le finalità dei diritti fondamentali, che assicurano la connessione del processo politico con le datità sociali. La giurisdizione costituzionale appare allora (...) quale parte istituzionalizzata, secondo le forme giudiziarie (gerichtsförmig), del processo politico, per così dire quale potere giudiziario allinterno della politica. (...) L'istituzionalizzazione della giurisdizione costituzionale è un processo di differenziazione, che presenta due lati. Da una parte ha luogo nel processo politico e rispetto ad esso, dall'altra all'interno e rispetto alla giurisprudenza. La giurisdizione costituzionale viene elevata al piano di attenzione con cui si segue la politica, e anche la selezione e lo status dei giudici vengono curati in maniera particolare. Tale giurisdizione diviene con ciò un po' più politica, ma viene al tempo steso neutralizzata per via istituzionale rispetto alla pressione esercitata da altri organi costituzionali. Ciò è del tutto chiaro nel caso della Supreme Court negli USA, ma vale anche per il Bundesverfassungsgericht [E. W. Böckenförde, 2006, 642-643].

Come si risolve dunque il dilemma della legittimazione democratica di attore dal ruolo così significativo e tanto penetrante in seno alle dinamiche dello Stato contemporaneo? Insufficienti appaiono a Böckenförde le garanzie offerte dalle modalità di elezione, nomina e durata del mandato dei giudici costituzionali, restando comunque alla giurisdizione costituzionale l'"ultima parola" sull'interpretazione della costituzione:

anche una ben bilanciata interdipendenza di proposte, elezioni, nomine e durata dei giudici non implica e non può implicare alcun controllo sulla giurisdizione costituzionale, 
néle è lecito implicarlo. La giurisdizione costituzionale esercita un controllo, ma non viene a sua volta controllata. L'ufficio del giudice costituzionale, pertanto, non è solo libero da controlli, è anche un ufficio dispensato dal dovere di render conto di ciò che fa. Ora, ̀̀ possibile creare un vincolo democratico su di esso che tuteli il principio della sovranità popolare? [E. W. Böckenförde, 2006, 657].

Una possibile risposta al quesito prospettato viene individuata dall'Autore nell'istituto del rinvio ai rappresentanti del popolo di una legge dichiarata incostituzionale dall'organo di giustizia costituzionale e nella possibilità che l'assemblea rappresentativa la riapprovi con la maggioranza (qualificata) richiesta ai fini della revisione costituzionale, contemplata dalla Costituzione polacca fino al luglio 1999. In tal caso l"'ultima parola" resterebbe all'organo di rappresentanza popolare: si tratta peraltro di soluzione non esente da rilievi critici, concernenti in particolare la possibilità, che ne deriverebbe, di modificare la costituzione per il caso singolo senza procedere ad una formale revisione costituzionale, implicitamente ammettendo che non si dia alcuna limitazione alle modifiche costituzionali.

Sullo sfondo stanno dunque due diverse concezioni della costituzione: da una parte la costituzione come un ordinamento prestabilito (vorgegeben) nel suo contenuto di fondo, fin dal momento in cui è stato approvato, avente validità universale e duratura e accessibile solo in parte al legislatore che voglia modificare la costituzione (costituzione come limitazione della sovranità popolare); dall'altra la costituzione come ordinamento per intero approvato e sostenuto dal popolo, non come in qualche modo prestabilito, e quindi anche a disposizione del popolo e sul quale nessun'altro può mai avere l'"ultima" parola (costituzione come espressione della sovranità) [E. W. Böckenförde, 2006, 658].

Affinché la giurisdizione costituzionale funzioni, svolgendo in pieno il ruolo di protezione della costituzione che le è proprio, una parte essenziale della responsabilità spetta tuttavia, secondo l'Autore, agli stessi giudici costituzionali:

essi devono essere coscienti della particolarità del loro compito, dei vincoli e anche dei limiti che sono connessi alla loro funzione, e devono adattarsi a questi vincoli e a questi limiti. Alle istanze, prevalentemente politiche, che trasmettono ai giudici la loro necessaria legittimazione democratica e la loro autorevolezza compete invece la responsabilità di nominare giudici solo persone all'altezza delle particolari esigenze che sono proprie di tale funzione giudiziaria, e sin dall'inizio immuni dalla tentazione di servirsi di tale ufficio non per far valere e salvaguardare la cornice giuridica per la politica, bensì per fare politica con altri mezzi, i mezzi cioè dell'interpretazione costituzionale. L'una e l'altra responsabilità non sono né delegabili né sostituibili; entrambe devono essere assunte liberamente. Anche da questo, e non per ultima cosa, dipende se la giurisdizione costituzionale divenga ciò che essa può e deve essere [E. W. Böckenförde, 2006, 659-660]. 


\section{g) approccio sistemico}

Paradigmatico e preliminare ai fini della ricostruzione dell'approccio sistemico è il classico insegnamento di O. Bachof [O. Bachof, 1959].

Per Bachof, un tratto caratteristico della Legge fondamentale, che la differenzia non solo dalle Costituzioni tedesche anteriori, ma anche da molte Costituzioni coeve, è dato dalla funzione assegnata al potere giudiziario in seno al sistema costituzionale. Tale elemento di novità è costituito dalla "funzione di controllo che la nostra Legge fondamentale ha riconosciuto al giudice di fronte agli altri "poteri" dello Stato, l'Esecutivo e il Legislativo" e per la straordinaria estensione di tale funzione di controllo. Tale incremento della funzione giudiziale implica un aumento accentuato dei poteri del giudice e, necessariamente, una diminuzione proporzionale del potere del Legislativo e dell'Esecutivo. È un fatto indiscutibile" [O. Bachof, 1959, 27].

L'accennata espansione delle attribuzioni del potere giudiziario, ed in particolare del Tribunale costituzionale federale, che nell'ordinamento tedesco rientra nella sfera del potere medesimo, è testimoniata secondo l'Autore dalla introduzione del controllo di legittimità costituzionale delle leggi, del ricorso diretto di costituzionalità a tutela dei diritti fondamentali (Verfassungsbeschwerde), della risoluzione dei conflitti interorganici ed intersoggettivi. Tali competenze non sono tuttavia in grado per se di giustificare l'efficacia pratica del controllo esercitato dal potere giudiziario: l'efficacia stessa è frutto della

energica ambizione di validità delle norme materiali della nostra Costituzione; di un ordine di valori che vincola direttamente i tre poteri statali e si manifesta espressamente nella disciplina dei diritti fondamentali; di un ordine di valori che -non nei suoi particolari, ma nelle norme fondamentali che lo costituiscono e lo legittimano- ̀̀ stato considerato dalla Costituzione come anteriore alla stessa; di un ordine di valori, dunque, che non è stato creato dalla Costituzione, ma che questa si limita a riconoscere e garantire ed il cui fondamento ultimo di validità si rinviene nei valori determinanti della cultura occidentale, in una concezione dell'uomo che si fonda sui tali valori [O. Bachof, 1959, 39-40].

Ciò spiega perché i diritti fondamentali, in quanto espressione dominante di tale ordine di valori, non possano essere modificati se non mediante una revisione formale del testo costituzionale e non potranno divenire oggetto di alcuna variazione ove ne risulti pregiudicato il valore fondamentale della dignità umana che li legittima: il nucleo istituzionale dei diritti fondamentali è garantito attraverso l'inviolabilità assoluta del loro contenuto sostanziale. Richiamando la formulazione già impiegata da H. Krüger [H. Krüger, 1950, 12], Bachof afferma che "in passato i diritti fondamentali valevano solo nell'ambito della legge, oggi le leggi 
valgono solo nell'ambito dei diritti fondamentali” [O. Bachof, 1959, 41]. I diritti fondamentali sono pertanto diritto immediatamente applicabile vincolanti, oltre che per la pubblica amministrazione, per lo stesso legislatore.

Quanto affermato con riferimento ai diritti fondamentali e relativamente all'ordine etico di valori ed alle sue pretese di validità immediata, risulta del resto applicabile, ad avviso del costituzionalista tedesco, al sistema politico in senso stretto: per la decisione adottata dalla Costituzione a favore della democrazia e della forma di governo parlamentare, a favore della separazione dei poteri come principio dello Stato di diritto per impedire abusi nell'esercizio del potere, a favore dei principi dello Stato sociale e dello Stato federale rispetto alla articolazione delle competenze fra i diversi organi statali [O. Bachof, 1959, 31].

La sostanza materiale della Costituzione così individuata, l'ordine di valori complessivamente considerato che la caratterizza, riposano operativamente sulla posizione dei tribunali: ai giudici è stata attribuita la responsabilità della protezione ultima della Costituzione. La ragione fondamentale della sottoposizione del legislatore al controllo dei giudici deve rintracciarsi, secondo Bachof, nel totale mutamento che ha interessato il rapporto tra gli uomini e la legge: è sorto un sentimento di profondo malessere e di radicale sfiducia nei confronti della legge, fondata sull'impressione dominante che la legge stessa, un tempo scudo della libertà e del diritto, si sia trasformata in una minaccia per tali beni [O. Bachof, 1959, 48]. La legge ha cessato di configurarsi quale norma generale ed astratta disciplinante i comportamenti umani, una ratio tradotta in norma, un mandato orientato alla giustizia, per convertirsi, nel moderno "Stato sociale", "Stato delle prestazioni", "Stato distributivo", in "un atto di conformazione politica orientato a un fine, come una misura determinata per superare una situazione totalmente concreta e, per tale motivo, pianificata a corto raggio e negoziata spesso nel conflitto di gruppi contrapposti di interessi” [O. Bachof, 1959, 51]. Le leggi sono divenute pertanto atti di indirizzo politico espressione di una volontà di natura politica condizionata dalle fattispecie contingenti e da circostanze occasionali. Si tratta di fenomeno evolutivo che ha portato la lotta per la preminenza dei singoli interessi concreti a lambire gli organi legislativi, diminuendo l'attitudine di tali organi a prendere in considerazione il valore giuridico che deve vincolare la volontà politica: per Bachof è sviluppo inevitabile che richiede necessariamente un contrappeso:

una forza che si preoccupi del fatto che, almeno, i valori superiori del diritto e l'ordine che la Costituzione ha stabilito come fondamentali rimangano protetti; una forza che decida, allo stesso tempo, con la maggiore autorità possibile, se in un eventuale conflitto quei valori sono stati oggetto di salvaguardia, assicurando o ristabilendo la pace giuridica [O. Bachof, 1959, 52]. 
Alle obiezioni mosse alla funzione di controllo attribuita all'organo di giustizia costituzionale -violazione del principio di separazione dei poteri, antidemocraticità insita nella decisione sul sistema costituzionale dei valori affidata ad un organo a composizione ristretta anziché al Parlamento, pericolo di politicizzazione della giustizia- l'Autore risponde attraverso un triplice ordine di considerazioni.

Quanto alla prima obiezione, Bachof considera che il significato della separazione dei poteri consiste nell'impedire la concentrazione del potere ed un possibile abuso dello stesso. Attualmente, nello Stato sociale moderno, il carattere necessario che ha per gli uomini, la quasi totale dipendenza di questi ultimi dall'apparato statale, implica correlativamente un controllo totale di tale apparato: la limitazione di potere che sperimentano Parlamento e Governo presuppone una correzione necessaria per il ristabilimento dell'equilibrio [O. Bachof, 1959, 58].

Relativamente al supposto carattere antidemocratico del potere giudiziario, l'Autore rileva, da un lato, che altri organi o soggetti non si trovano in un rapporto di diretta derivazione dal popolo (il Governo, il Presidente della Repubblica, i funzionari amministrativi), dall'altro che criterio di riferimento deve essere non il tipo di mandato ma piuttosto la funzione svolta: amministrare la giustizia in nome del popolo significa per il costituzionalista tedesco mantenere da parte del giudice un dialogo costante e continuo con le parti processuali, con i colleghi, con il mondo tecnico-giuridico e scientifico e con la pubblica opinione [O. Bachof, 1959, 60].

Sul versante dei rischi di politicizzazione dei giudici, infine, osserva l'Autore che il giudice esperto conosce i pericoli derivanti dalla introduzione nei suoi giudizi di elementi emozionali e irrazionali: la sua formazione professionale, la necessità di un dialogo ininterrotto e la stessa indipendenza del giudice garantiscono secondo tale visione un elevato livello di obiettività.

Il pericolo concreto che l'organo di giustizia costituzionale scivoli verso un paternalismo giudiziale che potrebbe implicare la transizione dallo Stato legislativo parlamentare ad uno Stato caratterizzato dalla preminenza della potestà giurisdizionale del Tribunale costituzionale è rischio che può evitarsi, secondo R. Alexy [R. Alexy, 1986, 526 ss.], mediante la incorporazione con successo della giurisdizione costituzionale nel processo democratico. Ciò presuppone che il Tribunale costituzionale sia concepito quale istanza di riflessione del processo politico e sia accettato come tale. A tal fine, l'organo di giustizia costituzionale deve esigere, in negativo, che il risultato del processo politico non contraddica i parametri dei diritti fondamentali e deve fondare, in positivo, le proprie pretese affinché i cittadini possano approvare razionalmente le argomentazioni del Tribunale. I diritti fondamentali devono interpretarsi, secondo il costituzionalista tedesco, in conformità ad una "concezione morale pubblica" che renda manifesta una rappresentazione 
comune delle condizioni giuste di cooperazione sociale in un mondo caratterizzato dal pluralismo: l'organo di giustizia costituzionale non deve contrapporre il proprio pensiero, in modo preconcetto, a quello del legislatore, ma aspirare a configurarlo come una rappresentazione argomentativa dei cittadini.

La Costituzione prescrive e vieta certe azioni attraverso le norme fondamentali ed i principi formali stabilendo un ordinamento-quadro quale ordine fondamentale; lascia aperti, peraltro, margini di manovra, strutturali ed epistemici, attribuendo una certa fiducia al legislatore ai fini della concretizzazione dei diritti fondamentali. Analogamente, l'intervento dell'organo di giustizia costituzionale, quando constata una violazione della competenza da parte del legislatore, non produce uno spostamento istituzionale della competenza del Tribunale nell'ambito della legislazione. Tale intervento, segnala Alexy, non solo è permesso, ma è prescritto dalla Costituzione, nella misura in cui la stessa ne determina la sfera d'azione. Il nucleo essenziale di tale sfera di attribuzioni consiste nella protezione di un ordinamento-quadro che stabilisce simultaneamente un ordine fondamentale [R. Alexy, 1986, 529].

La considerazione delle funzioni dell'organo di giustizia costituzionale nella più generale dinamica sociale è criterio seguito dagli Autori che hanno riservato alla problematica della legittimazione della giurisdizione costituzionale un approccio sistemico, imperniato sul riferimento a criteri giuridico-costituzionali, ma fondato altresì sul ricorso a criteri di natura lato sensu sociale e politica, economica, storica e culturale.

Ne è un esempio la ricostruzione di E. Stein [E. Stein, 1972, 485 ss.], che identifica sette funzioni del Tribunale costituzionale federale in seno al sistema politico tedesco. Secondo tale posizione dottrinale, l'organo di giustizia costituzionale è chiamato a svolgere una funzione di stabilizzazione della Costituzione, agevola contestualmente un mutamento sociale controllato (funzione evolutiva), fungendo anche da valvola capace di prevenire (od ovviare a) fenomeni di ristagnamento delle forze politiche e sociali (funzione-valvola). In quanto organo di controllo competente a verificare che il processo di formazione della volontà politica si svolga all'interno dell'alveo e dei limiti ad esso assegnati, il Tribunale costituzionale federale corregge in modo vincolante comportamenti contrastanti con la Costituzione (funzione di controllo). Il componimento dei conflitti realizzato dall'organo di giustizia costituzionale contribuisce alla formazione della pace sociale e risolve conflitti politici e sociali mediante una procedura di tipo giurisdizionale (funzione di pacificazione). L'attuazione dei diritti fondamentali promuove un'attivazione della libertà (funzione educativa) e la conservazione del carattere aperto del processo politico, in particolare attraverso la protezione delle minoranze, consente di superare crisi di legittimazione del sistema politico, portando alla realizzazione della democrazia (funzione di integrazione). 
Accanto a posizioni dottrinali, quale quella ora esaminata, tendenti a conferire una pluralità di compiti e funzioni alla giustizia costituzionale, altre hanno inteso individuarne finalità più specifiche e mirate.

In un'ottica di critica al modello capitalistico, O. Massing [O. Massing, 2005, 81 ss.] configura la giurisdizione costituzionale quale componente centrale del sistema di governo politico-amministrativo dello Stato interventista tardo-capitalistico, dotata della funzione di sviluppare e conservare l'equilibrio e la stabilità internamente al sistema medesimo. Il Tribunale costituzionale federale, in quanto strumento di creazione del consenso in modo vincolante e di influsso sugli imperativi di azione collettivi, viene concepito dall'Autore quale fattore di gestione della crisi politica dello Stato tardo-capitalisitico e di sostegno ad un sistema di forze strutturato secondo un modello oligopolitistico.

Analoghe le argomentazioni addotte da R. Schlothauer [R. Schlothauer, 1979], che identifica la crisi della giurisdizione costituzionale con una crisi della Costituzione. Rilevanza determinante viene attribuita in tale direzione alla "crisi dei metodi del Tribunale costituzionale federale", intesa quale poliedricità e qualunquismo dei metodi interpretativi dell'organo di giustizia costituzionale che darebbe origine ad un "caos metodologico" tale da ingenerare la totale perdita di legittimazione delle singole pronunce. Ad avviso dell'Autore, i criteri metodologici utilizzati dall'organo di giustizia costituzionale sono finalizzati al raggiungimento di due scopi essenziali: la depoliticizzazione dei conflitti di interesse in seno alla società ed il camuffamento delle loro cause all'interno della sfera legislativa. E per tali ragioni che il Tribunale costituzionale federale non sarebbe in grado di legittimare l'esercizio di attività sovrane, ma semplicemente di mascherarlo: secondo Schlothauer, la crisi di legittimazione della sovranità non viene risolta, ma solo rinviata.

In un'ottica di critica ideologica parallela alla precedente, lo studio di K.-H. Ladeur [K.-H. Ladeur, 1980, 189 ss.] si incentra sul nesso della giurisdizione costituzionale con la "normalità" sociale. L’Autore sviluppa tale concetto sulla base del paradigma della "convenzione fondamentale" della società civile che in una democrazia liberaldemocratica lega la legittimazione di ogni legge al consenso del soggetto titolare della sovranità attraverso un processo decisionale democratico. Le modalità funzionali di Parlamento ed Esecutivo sono state coordinate secondo Ladeur mediante una convenzione fondamentale, che potrebbe presupporre nel suo complesso "la Costituzione-contratto sociale quale modello funzionale relativamente solido di 'normalità' civile". Nella transizione al capitalismo caratterizzato dall'interventismo statale i presupposti di tale modello funzionale sono ora venuti meno: il soggetto di diritto quale entità di riferimento per l'azione statale si è liquefatto, i suoi comportamenti e le sue esigenze non rappresentano più una 
misura calcolabile, ma necessitano della pianificazione, la modalità funzionale del consenso si è separata dal procedimento legislativo e si riferisce ora al sistema politico nel suo complesso: tali caratteristiche sono secondo Ladeur un segnale della perdita di identità dello Stato quale oggetto universale e della decadenza e degenerazione dell'ordinamento borghese. Dalla diversa sostanza della legge derivano conseguenze anche per la giurisdizione costituzionale: la disintegrazione del principio della generalità e l'accentuazione della contingenza della legge implicano che la medesima sia sormontata da un simbolo astratto, la Costituzione, sul quale vigila l'organo di giustizia costituzionale, le cui funzioni principali consistono nel controllo e nella unificazione della formazione delle ideologie dominanti, nonché nella realizzazione della coerenza ideologica delle strategie degli apparati statali. Secondo Ladeur il Tribunale costituzionale federale adempie il suo compito principale nell'arena della constituent policy: positivamente mediante la cura dei simboli e la elaborazione del consenso, negativamente attraverso la mancata tematizzazione di problematiche fondamentali come la redistribuzione, nonché tramite la stigmatizzazione di "nemici antisistema" a garanzia dello status quo politico ed economico.

Analogamente, secondo U. K. Preuss la funzione cardine del Tribunale costituzionale federale consiste nella formulazione di un "consenso di fondo" (Grundkonsens) quale meta-diritto, alle cui condizioni la Costituzione soddisfa la propria funzione di regolazione sociale [U. K. Preuss, 1987, 1 ss.].

La concezione integrazionistica della giurisdizione costituzionale ha sempre rivestito una primaria importanza, quanto meno a partire dalla teoria di R. Smend sulla forza integrativa della Costituzione [R. Smend, 1928]. Più recentemente, le teorie pluralistiche della giustizia costituzionale sono divenute oggetto di riconsiderazione, in particolare, in seno alle posizioni dottrinali di P. HÄberle e di I. Ebsen.

L'inquadramento della giustizia costituzionale avviene nelle opere di P. Häberle, sia di carattere generale [P. Häberle, 2005] che di contenuto specificamente dedicato alla materia in oggetto [P. Häberle, 1980 e 2006, 35 ss.], alla luce del concetto di Costituzione:

"Costituzione" è l'ordinamento giuridico fondamentale di stato e società; essa non è solo limite del potere statale, ma anche autorizzazione al potere statale; essa racchiude Stato e società. La giustizia costituzionale come forza politica agisce sin dal principio al di fuori del dogma della divisione Stato/società [P. Häberle, 2005, 158].

La configurazione del Tribunale costituzionale federale quale "corte costituzionale" di tutta la res publica implica che l'organo di giustizia costituzionale non aderisca ad una teoria o scuola dottrinale, ma persegua l'integrazione pragmatica di elementi di diverse teorie. 
Questo riferimento alla costituzione materiale della giustizia costituzionale determina rilevanti implicazioni sostanziali e processuali: ad es. l'obbligo della Corte di operare verso il modello del pluralismo e nell'incentivazione della costruzione del diritto costituzionale processuale con riguardo a pluralistici strumenti di informazione e partecipazione [P. Häberle, 2005, 159].

Il pluralismo nelle procedure costituzionali

costituisce una condizione necessaria per la guida della società da parte della corte costituzionale e del diritto da questa elaborato. In tale ambito si giunge così ad uno scambio reciproco di influenze: tanto più la Corte costituzionale federale interviene nei processi di guida della società aperta, tanto più la società si rivolge ad essa. (...) Questa impostazione conduce inoltre ad un nuovo "livello", al quale la Corte costituzionale federale deve essere considerata nel suo stretto legame con la società in generale: essa diviene una "corte sociale" sui generis ed in senso lato. Attraverso la sua giurisprudenza essa si apre alla molteplicità delle idee e degli interessi-li assume in sé-e a contrario guida la società [P. Häberle, 2005, 159].

La concezione del Tribunale costituzionale federale più come "corte della società in generale" che come corte "statale" implica il conferimento in capo all'organo di giustizia costituzionale di

una peculiare responsabilità generale nella garanzia e nello sviluppo della costituzione come contratto sociale, incidendo sul suo processo continuo di realizzazione ed è in ciò obbligato verso il pluralismo. (...) La funzione della giustizia costituzionale è la limitazione, la razionalizzazione e il controllo del potere statale e sociale, essa (co)opera, dal punto di vista del contenuto, al consenso generale e si incentra nella tutela delle minoranze e dei deboli, nella reazione flessibile e tempestiva ai nuovi pericoli per la dignità dell'uomo, nel suo carattere a-politico di guida e risposta [P. Häberle, 2005, 159-160].

Analogamente, secondo la teoria della giustizia costituzionale elaborata da I. Ebsen, nello Stato costituzionale democratico vengono attribuiti agli organi di giustizia costituzionale tre compiti fondamentali: conservazione dell'apertura del processo politico, che presuppone la legittimità del principio maggioritario; garanzia della misura socialmente consentita di protezione dell'individuo nei confronti della forza statale; integrazione degli interessi particolari nella misura tale da rendere possibile il sistema decisionale maggioritario [I. Ebsen, 1985, 229]. I tre compiti indicati presuppongono secondo l'Autore un determinato grado di consenso: la funzione preordinata al mantenimento del carattere aperto del processo politico si fonda sul consenso al meta-livello dei rapporti sociali; la funzione normativa di tutela della libertà è riconducibile agli elementi del concetto generale di libertà, apportati in linea di principio dal consenso costituzionale materiale; il consenso, che da una parte presuppone la funzione di integrazione, ma dall'altra è finalizzato a garantire, 
concerne un ulteriore meta-livello connotato dall'obiettivo di garantire ed attuare il compromesso sociale formatosi in sede costituente [I. Ebsen, 1985, 322].

\section{II) L'approccio filosofico}

Fra le posizioni dottrinali che si caratterizzano per un approccio filosofico alla problematica della legittimazione della giustizia costituzionale conviene soffermarsi su quelle elaborate da O. Höffe e da J. Habermas.

Höffe constata [O. Höffe, 1996; O. Höffe, 1995] una perdita di fiducia nel Tribunale costituzionale federale tedesco, che l'organo di giustizia costituzionale avrebbe causato attraverso una serie di pronunce. Lo studio dell'Autore si incentra su due profili principali: da una parte la funzione del Tribunale di garanzia e sviluppo del consenso, dall'altra l'equilibrio delle forze in seno al sistema democratico. La legittimità della giurisdizione costituzionale, in particolare, dipende - secondo la posizione dottrinale in oggetto - dalla diversa proporzione assegnata ai pesi dei pilastri dello Stato costituzionale democratico (i sistemi politici che si fondano sul principio di separazione dei poteri si reggono sulla sovranità popolare e sulla democrazia, nonché sul legame con i principi e valori costituzionali, in particolare i diritti fondamentali e la separazione dei poteri): se si concepiscono i principi costituzionali in un senso meramente giuridico-morale, il potere supremo risiede presso la rappresentanza popolare; se al contrario tali principi vengono intesi in senso giuspositivistico, la Costituzione tende ad acquisire la forza suprema. Dalla vincolatività giuridica dei principi giuridico-costituzionali deriva un incremento della forza del potere giudiziario, poiché la giurisprudenza -in quanto giurisdizione costituzionale- acquisisce un potere di controllo sugli altri poteri [Höffe, 1996, 258]. L'autore non si misura con tale alternativa, ma si limita ad osservare che i due pilastri dello Stato costituzionale democratico si trovano in un rapporto di reciproca tensione. L'istituzione della giurisdizione costituzionale non risulta indefettibile dal punto di vista della teoria democratica: nota Höffe, a tale riguardo, come non tutti gli Stati democratici si siano dotati di un sistema di giustizia costituzionale e che, laddove istituito, tale sistema non appare così ampio ed articolato, quanto alle competenze contemplate, come quello tedesco. Il ruolo della giurisdizione costituzionale nel concerto dei poteri pubblici deve considerarsi alla luce del principio della correlazione antiproporzionale fra intensità di impatto politico ed entità delle competenze: il controllo costituzionale deve essere esercitato in misura inversamente proporzionale rispetto all'entità quantitativa e qualitativa delle competenze attribuite all'organo di giustizia costituzionale.

"Troppo espansiva" appare all'Autore, in particolare, la giurisprudenza del Tribunale costituzionale federale che tende ad estendere la tutela dei diritti fon- 
damentali ai presupposti reali di realizzazione dei diritti medesimi, riconoscendo a questi ultimi, accanto ad una dimensione soggettiva, una dimensione oggettiva: il ruolo dell'organo di giustizia costituzionale si traduce in tali casi in una seconda interpretazione di controllo (kontrollierende Zweitinterpretation) capace di facilitarne l'intromissione nel dominio riservato al legislatore [Höffe, 1996, 270].

Il pensiero di Habermas in materia si snoda essenzialmente in seno a due fondamentali opere [J. Habermas, 1998 e 1996, in particolare cap. VI] e adotta come premessa essenziale la differenziazione fra concezione "liberale" e concezione "repubblicana" ai fini della individuazione del ruolo che si intende attribuire al processo democratico:

Secondo la concezione "liberale" (...) tale processo serve a programmare lo Stato nell'interesse della società, laddove per Stato sintende l'apparato dell'amministrazione pubblica e per società il sistema di commercio degli individui privati (strutturato dall'economia di mercato) e il sistema del loro lavoro sociale. In questa prospettiva la politica - intesa come formazione politica della volontà dei cittadini - ha la funzione di aggregare e far valere gli interessi sociali dei privati nei confronti dell'apparato statale, mentre quest'ultimo si specializza nell'uso amministrativo del potere politico per obiettivi comuni. Secondo la concezione repubblicana, invece, la politica non si riduce a questa funzione di mediazione, ma è piuttosto costitutiva del processo di socializzazione nel suo insieme. Per "politica" si intende allora la forma di riflessione di un vitale nesso etico, cioè il medium attraverso cui individui organicamente inseriti in comunità più o meno naturalmente solidali-facendosi consapevoli della loro reciproca dipendenza-perfezionano e sviluppano con volontà e coscienza, come cittadini dello Stato, i rapporti ereditati di riconoscimento reciproco, trasformandoli in un'associazione di liberi ed eguali consociati giuridici. Così l'architettura liberale del rapporto Stato-società subisce unimportante modifica: accanto alla (gerarchizzata) istanza regolativa del potere statale e alla (decentralizzata) istanza regolativa del mercato -dunque accanto a potere amministrativo e interesse egoistico privato- vediamo affacciarsi solidarietà e orientamento al bene comune come terza fonte dell'integrazione della società [J. Habermas, 1996, 319, grassetti nostri].

Dai due approcci concorrenti deriva una serie di conseguenze ai fini della valutazione del processo politico sui versanti del concetto di cittadinanza e di diritto; la concezione repubblicana della politica sottolinea il nesso interno tra sistema dei diritti e autonomia politica dei cittadini:

da questa prospettiva la Corte costituzionale, nel quadro delle sue competenze, deve far sì che il processo della produzione del diritto si compia nelle condizioni legittimanti d'una politica deliberativa. Questultima è per parte sua legata agli esigenti presupposti comunicativi di arene politiche non circoscritte alla formazione istituzionalizzata della volontà dei corpi parlamentari, ma estese anche alla più ampia sfera pubblica, nonché al suo contesto culturale e base sociale. Una prassi discorsiva di autodeterminazione può 
soltanto svilupparsi nel gioco di scambio che sinstaura tra la formazione di volontà dei corpi parlamentari-istituzionalizzata da regole procedurali e programmata per decidere-e quella formazione politica dell'opinione che avviene nei circuiti informali della comunicazione politica. Iniziative, temi e contributi, problemi e proposte di rilievo giungono più spesso dai margini dell'arco delle opinioni piuttosto che dal suo centro consolidato [J. Habermas, 1996, 326-327].

La lettura vitalistica che Habermas offre dell'autodeterminazione democratica tende a giustapporre la volontà popolare, "sonnecchiante in lunghi periodi di latenza”, e l'attività legislativa istituzionalizzata dei rappresentanti eletti:

durante questi lunghi intervalli i giudici della Corte costituzionale -custodi d'una prassi d'autodeterminazione momentaneamente congelata nella routine del negozio parlamentare- devono utilizzare in maniera vicaria i diritti d'autodeterminazione del popolo. (...) La Corte costituzionale diventa il luogotenente repubblicano per quelle libertà positive che i cittadini - benché titolari nominali di esse-sono incapaci di esercitare personalmente. (...) È soltanto questa descrizione eccezionalista della prassi politica-come quella che dovrebbe essere se fosse genuina -ciò che rende alla fine necessaria la presenza d'un luogotenente pedagogico. Questi deve servire soltanto nei periodi di reggenza, ossia per tutto il tempo in cui il sovrano preferisce rinchiudersi nella dimensione privata, anziché occupare come gli compete la sfera pubblica e lì esercitare $i$ suoi doveri. Questimmagine "eccezionalista" di quel che dovrebbe essere la politica dipende in realtà da una tradizione repubblicana che ha sempre ricondotto la prassi politica dei cittadini all'ethos d'una comunità fin dall'inizio integrata [J. Habermas, 1996, 330-331, grassetti nostri].

Dalla concezione procedurale della Costituzione derivano nella ricostruzione dell'Autore due corollari. In primo luogo, la giurisdizione costituzionale è concepita quale strumento finalizzato a rendere chiaro il diritto, a garantire la coerenza dell'ordinamento e ad individuare il surplus di diritto (realizzandolo nelle proprie decisioni) esistente rispetto alle statuizioni positive del potere statale, un diritto non coincidente con l'insieme delle leggi scritte che ha la sua fonte nell'ordinamento giuridico costituzionale inteso come totalità e che può fungere da correttivo della legge scritta.

Assumendo come esempio il Tribunale costituzionale federale tedesco, l'Autore distingue tre ambiti di competenze: i conflitti interorganici ed intersoggettivi, il controllo di legittimità costituzionale delle leggi, i ricorsi diretti di costituzionalità posti a difesa dei diritti fondamentali. Secondo Habermas, non sorgono particolari problemi, sul versante della separazione dei poteri, con riferimento ai ricorsi individuali ed al controllo concreto di legittimità costituzionale esercitato in via incidentale, funzionando in tali casi l'organo di giustizia costituzionale quale garante dell'unità dell'ordinamento giuridico: 
la concorrenza tra Corte costituzionale e legislatore democratico legittimo si acutizza seriamente soltanto nell'ambito del controllo astratto di legittimità, quando cioè si sottopone a verifica giudiziaria la questione se una certa legge licenziata dal parlamento sia conforme alla costituzione e non contraddica quanto meno un organico sviluppo del sistema dei diritti [J. Habermas, 1996, 287-288].

Il secondo corollario concerne la concezione della Costituzione ed il ruolo spettante al legislatore ed alla giurisdizione costituzionale in sede di sviluppo della medesima: il vincolo di entrambi gli organi ai dati procedurali della Costituzione non implica alcuna assimilazione concorrenziale tra i medesimi:

alla Corte costituzionale le ragioni legittimanti (desumibili dalla costituzione) vengono date nella prospettiva dell'applicazione giuridica, non nella prospettiva d'un legislatore che interpreta e sviluppa il sistema dei diritti perseguendo indirizzi di "policy". La Corte riapre il fascicolo di ragioni già "confezionato" dal legislatore nella legittimazione delle sue delibere, e mobilita nuovamente queste ragioni per decidere il singolo caso in maniera coerente e sintonizzata ai principi giuridici vigenti. Ma di queste ragioni la Corte non può disporre liberamente per interpretare e sviluppare in sede immediatamente giudiziaria il sistema dei diritti (ciò che instaurerebbe una sorta di legislazione implicita) [J. Habermas, 1996, 311-312].

La Costituzione non è più pensabile come "ordinamento quadro" disciplinante unicamente o prevalentemente il rapporto dell'amministrazione con i cittadini, né come un ordinamento giuridico globale e completo che cali dall'alto sulla testa della società una determinata forma di vita:

la costituzione stabilisce piuttosto dei procedimenti politici in base ai quali i cittadini -esercitando il loro diritto di autodeterminazione- possono perseguire in forme cooperative e con speranza di successo il progetto di creare rapporti di vita giusti (il che significa: sempre più giusti). Solo le condizioni procedurali relative alla genesi democratica delle leggi assicurano legittimità al diritto positivo. Prendendo le mosse da questa "concezione di fondo" democratica possiamo dare anche alle competenze della Corte costituzionale un senso che corrisponda allintenzione costituzionale della divisione dei poteri: alla Corte costituzionale tocca proteggere il sistema dei diritti che rende possibile l'autonomia privata e pubblica dei cittadini. (...) Perciò la Corte costituzionale dovrà controllare le norme controverse avendo soprattutto riguardo ai presupposti comunicativi e alle condizioni procedurali della legislazione democratica. Questa concezione proceduralistica della costituzione dà una svolta in senso democratico alla questione della legittimità della giustizia costituzionale [J. Habermas, 1996, 312-313].

Il controllo astratto di legittimità costituzionale dovrà riguardare in primo luogo, in tale ottica, le condizioni della genesi democratica delle leggi:

qui l'esame deve partire dalle strutture comunicative d'una sfera pubblica manipolata dai mass-media, passare alle possibilità effettive che le voci dissenzienti hanno di farsi 
sentire e di far valere $i$ loro diritti (formalmente eguali) di partecipazione, giungere poi alla rappresentazione paritaria che i corpi parlamentari offrono di tutti i gruppi, posizioni d'interesse e orientamenti di valore via via rilevanti, e infine concludersi sul ventaglio dei temi, ragioni e problemi, valori e interessi, che dopo aver trovato accesso nelle consultazioni parlamentari sono tenuti in conto nella fondazione delle norma deliberate [J. Habermas, 1996, 314].

\section{III) L'approccio politologico}

Fra le posizioni dottrinali che si caratterizzano per un approccio politologico alla problematica della legittimazione della giustizia costituzionale è doveroso ricostruire sinteticamente quelle elaborate da C. J. Friedrich e da H. Vorländer.

C. J. Friedrich ha affrontato la problematica in esame in seno al capitolo XII ("La revisione giudiziaria degli atti legislativi; la tutela della Costituzione") di un'opera classica della letteratura politologica [C. J. Friedrich, 1963, 318 ss., cui adde C. J. Friedrich, 2002, 177 ss.].

Individuati quali fattori di rafforzamento della judicial review l'idea di una Costituzione che concretizza il diritto più alto della legislazione ordinaria e l'idea del federalismo, Friedrich individua tre gradi di interpretazione politica della funzione costituzionale e politica di una corte suprema:

(1) la corte interpreta la costituzione, essendo le norme della costituzione chiare come un'equazione matematica (opinione popolare); (2) la corte è uno strumento della politica dei partiti, essa decide secondo le opinioni politiche dei giudici, è antidemocratica (opinione politica); (3) la corte è l'alto sacerdozio della fede nel costituzionalismo, essa razionalizza la nuova norma in termini della vecchia e con ciò mantiene la continuità, se non la coerenza; in breve, essa arbitra fra le fondamentali e sempre presenti forze rivali in un sistema costituzionale. E questo terzo grado di interpretazione che riconosce pienamente la funzione di un'alta corte come interprete e tutrice dei sacri simboli di parole che tengono assieme molti uomini e molte menti in una comunità organizzata [C. J. Friedrich, 1963, 325].

L'attitudine disinteressata dei giudici che compongono gli organi di giustizia costituzionale e la fede della comunità in tale obiettività vengono individuati dall'Autore quali fattori fondamentali ai fini del riconoscimento del carattere veramente rappresentativo degli organi medesimi: è alla luce di tali considerazioni che Friedrich muove la propria critica verso la tendenza degli europei a rendere le loro nuove corti costituzionali o elettive da parte del corpo legislativo o addirittura comitati del corpo legislativo. La divisione in partiti di quest'ultimo è in grado di minarne la qualità rappresentativa in misura significativa; d'altra parte, una corte composta da eminenti talenti giuridici può risultare in grado di rappresentare le convinzioni della comunità riguardo a principi di giustizia oggettiva ed universale: 
(...) la questione di quanto sia rappresentativo il potere giudiziario di una nazione, è una questione di fatto. Più profondamente è radicato il rispetto della comunità per i tribunali, meno pericolo esiste che questi assumano la funzione arbitrale circa la definitiva interpretazione costituzionale. Ciò è particolarmente vero se il procedimento di emendamento offre speranza agli scontenti di poter modificare le disposizioni della costituzione che danno loro fastidio. I tribunali non godono tale rispetto senza riserve, in quelle zone dell'Europa in cui il movimento marxista è forte. Infatti, secondo la dottrina della lotta di classe di Carlo Marx, i tribunali non sono altro che esponenti camuffati della classe borghese, che contribuiscono alla sfruttamento delle masse che si affaticano. In altre parole, essi amministrano la giustizia di classe, invece della giustizia di massa. E evidente che ogniqualvolta tali opinioni hanno larga diffusione, la fiducia generale nel "disinteresse" del potere giudiziario viene meno. Se in tali condizioni si chiede ai tribunali di decidere cause che comportano l"interpretazione" delle disposizioni costituzionali, che possono essere in parte socialiste e in parte no, le controversie risultanti mineranno ulteriormente la loro posizione, indipendentemente da come essi decidano. Se, inoltre, molti dei giudici sono trasmessi da un regime precedente, e mantenuti a causa del principio dell'esercizio delle funzioni giudiziarie durante un buon comportamento, la lealtà dei tribunali verso il nuovo governo può pure diventare sospetta [C. J. Friedrich, 1963, 336-337].

La tecnica politica della judicial review può dunque essere impiegata ad avviso dell'Autore solo ove il popolo nel suo complesso nutra una considerevole fiducia nell'integrità delle corti.

D'altra parte, nessuna "giuria costituzionale" diversa da tale potere giudiziario sarà sufficientemente neutrale e distaccata per esercitare efficacemente le funzioni di tutrice della costituzione. Dobbiamo concludere, quindi, che nell'assenza di una costituzione profondamente radicata nella tradizione, come esiste in Inghilterra, in Svizzera o in Svezia, sarà necessario un potere giudiziario capace di esercitare la revisione giudiziaria se una costituzione, nel senso politico di una serie di tecniche per limitare le azioni del governo, deve essere istituita. Soltanto attraverso l'influenza neutralizzatrice e razionalizzatrice di tale interpretazione giudiziaria i vari interessi, gruppi e classi nella comunità saranno mantenuti sufficientemente in equilibrio. Persino un siffatto potere giudiziario può esser in grado di adempiere tale funzione. E un sano segno del rafforzamento del costituzionalismo in Europa che le nuove costituzioni nell'Europa occidentale si sforzino tutte di istituire qualche genere di revisione giudiziaria degli atti legislativi. L'estensione di questi sforzi sembra sia in relazione inversa alla loro esperienza con il totalitarismo. Alla luce della storia dell'istituzione, pare abbastanza naturale che coloro che hanno risentito più intensamente dei risultati della perdita del costituzionalismo siano i più preoccupati di garantirlo contro la futura minaccia da parte delle forze costituzionaliste [C. J. Friedrich, 1963, 339].

Più recentemente, H. Vorländer [H. Vorländer, 2006, 9 ss.] ha individuato nell'organo di giustizia costituzionale, con specifico riferimento all'esperienza del 
Tribunale costituzionale federale tedesco ma sulla base di considerazioni suscettibili di generalizzazione, un elemento integrante del potere (Machtfaktor) nell'ambito del sistema politico, in seno al quale l'organo medesimo svolge non solo una funzione di mediazione ed arbitrale ai fini della risoluzione dei conflitti che sorgono nell'alveo della competizione politica, ma è anche divenuto attore politico.

Ma il Tribunale costituzionale non è solo un policy maker ed in quanto tale coinvolto nella competizione politica, politics. Forse ancora più significativo è il ruolo che la giurisdizione costituzionale gioca ai fini della concreta conformazione, anche in senso progressivamente modificativo dei fondamenti dell'ordinamento politico, dunque propriamente della polity. Da una parte essa assume a tale riguardo, mediando conflitti procedurali o materiali fra gli organi politici o le componenti federali, la funzione di custode dell'ordine costituzionale delle competenze e delle procedure. Dall'altra le corti costituzionali decidono in modo sostanziale sull'interpretazione ed applicazione dei diritti fondamentali e civili, sulle condizioni aggregative e relazionali della comunità politica, sugli spazi della libertà pubblica e della partecipazione politica, sui limiti del pubblico potere e sulle sfere di libertà privata dei cittadini [H. Vorländer, 2006, 11].

L'attività svolta dagli organi di giustizia costituzionale nelle direzioni ricordate contribuisce a collocarli in una posizione di forza (machtvoll), di primo piano (überragend), riconosciuta dal potere legislativo ed esecutivo e supportata dalla fiducia del popolo. La corte o tribunale costituzionale, bouche de la Constitution (der Mund der Verfassung), interprete definitivo della Costituzione, è organo posto in una posizione di preminenza che gli deriva dalla primazia della Costituzione medesima: con riferimento all'interpretazione di una Costituzione, l'aspetto di decisiva importanza consiste nella metamorfosi della competenza normativa originariamente esercitata dal costituente sovrano che diviene competenza interpretativa del soggetto chiamato ad interpretare la Costituzione [H. Vorländer, 2006, 14].

La "forza" dei tribunali costituzionali si evince empiricamente dal fatto che le istituzioni politiche e giudiziarie seguono l'interpretazione della giurisdizione costituzionale e che a tale interpretazione si conformano nella loro prassi concreta: gli organi di giustizia costituzionale non dispongono tuttavia di potestà sanzionatorie per ottenere in via coercitiva l'ottemperanza alle proprie decisioni:

una corte costituzionale non dispone di propri soldati o forze dell'ordine che possano costringere all'accettazione ed applicazione di una sentenza. Ne deriva che le decisioni possono sempre risultare vane, essere ignorate o trascurate od osteggiate dalle istituzioni politiche. Il potere della giurisdizione costituzionale non riposa pertanto sul rigido schema di comando e obbedienza [H. Vorländer, 2006, 16].

L'Autore identifica nel potere interpretativo delle corti costituzionali una specifica forma di potere che si fonda su valori simbolici e comunicativi e che si 
manifesta nell'attuazione di idee-guida ed esigenze fondamentali. Tali idee e valori sono "trasportati" dalle costituzioni dalla fase costituente al momento attuale, conservando la reminiscenza dell'atto fondativo dell'ordinamento politico ed assicurandone la perpetuazione nel tempo:

non esiste tuttavia alcun automatismo in sede di trasformazione della validità della fase fondativa, risultante dallo stesso atto costituente, nella validità attuale. Genesi e validità tendono a disgiungersi con la permanenza in vigore della costituzione. La forza vincolante della costituzione deriva dal mutamento della validità fondativa in validità permanente. Ma tale stabilizzazione della validità della costituzione significa contestualmente che il potere degli autori della costituzione viene trasformato in autorità degli interpreti della Costituzione. Al posto dell'autore subentra l'interprete [H. Vorländer, 2006, 19].

Al fine di individuare le ragioni giustificative dell'autorità e della legittimazione degli interpreti della Costituzione, Vorländer ripropone la distinzione già delineata da H. Arendt nel suo saggio Sulla rivoluzione che, sulla base dell'esperienza romana, differenziava l'auctoritas del Senato dalla potestas del popolo. Riferendosi alla fase costituente nell'esperienza nordamericana osserva l'Autrice che

fu l'esperienza (...) piuttosto che la teoria o la dottrina, che insegnò agli uomini della rivoluzione il vero significato della romana potestas in populo, ossia che il potere risiede ne popolo. Essi sapevano che il principio della potestas in populo è in grado di ispirare una forma di governo soltanto se vi si aggiunge, come fecero i romani, auctoritas in senatu, che l'autorità risiede nel senato; sicché il governo stesso consiste di potere e autorità, o, come dicevano i romani, senatus populusque Romanus [H. Arendt, 2006, 203-204].

Fra le numerose innovazioni che il nuovo assetto costituzionale lasciava trasparire,

la più importante forse, e certo la più cospicua, consisteva nello spostamento della sede dell'autorità dal senato (romano) al poter giudiziario del governo; ma ciò che rimase vicino allo spirito romano fu l'esigenza e l'instaurazione di una istituzione concreta la quale, ben distinta dalle competenze del potere legislativo e di quello esecutivo del governo, era espressamente destinata alla funzione di detenere l'autorità. (...) Dal punto di vista istituzionale, ciò che indica come la vera sede dell'autorità nella repubblica americana sia la Corte suprema è proprio la mancanza di potere, combinata con l'investitura permanente. E questa autorità viene esercitata in una specie di continua elaborazione della costituzione, dal momento che la Corte suprema è effettivamente, come diceva Woodrow Wilson, 'una specie di Assemblea costituzionale in seduta continua'.

Tuttavia, mentre la differenziazione istituzionale americana fra potere e autorità presenta distintamente caratteri romani, il concetto di autorità è qui del tutto diverso. A Roma la funzione dell'autorità era politica e consisteva nell'attività consultiva, mentre nella repubblica americana la funzione dell'autorità è giuridica e consiste nell'interpretazione. La Corte suprema deriva la sua autorità dalla Costituzione come documento scritto, mentre 
il senato romano, i patres o padri della repubblica romana, detenevano la loro autorità perché rappresentavano, o piuttosto reincarnavano, i progenitori la cui unica pretesa di autorità nello stato stava precisamente nel fatto che lo avevano fondato, che erano $i$ "padri fondatori". Nella persona dei senatori romani continuavano a essere presenti $i$ fondatori di Roma, e con loro era presente lo spirito della fondazione, il cominciamento, il principium e il principio di quelle res gestae che da quel momento in poi formarono la storia del popolo romano, giacchè l'auctoritas, la cui radice etimologica è augere, accrescere e aumentare, dipendeva dalla vitalità dello spirito di fondazione, in virtù del quale era possibile aumentare, accrescere e ampliare le fondamenta che erano state gettate dai progenitori. Lininterrotta continuità di questo accrescimento e l'autorità insita in esso potevano realizzarsi solo attraverso la tradizione, ossia attraverso la trasmissione, lungo una linea ininterrotta di successori, del principio stabilito allinizio [H. Arendt, 2006, 229-231].

È sulla base di tali considerazioni che Vorländer ritiene di potere identificare il potere interpretativo della giurisdizione costituzionale con l'autorità che deriva dall'atto costituente, potere che consente di mantenere vitali i principi e valori fondamentali dell'ordine politico, ma anche di svilupparli, adeguandoli alle congiunture. Con ciò la giurisdizione costituzionale viene sganciata dalla quotidianità politica, dai conflitti e dalle lotte di potere e riceve una dignità particolare, uno status quasi aristocratico nel sistema misto della democrazia costituzionale: la giurisdizione costituzionale possiede pertanto un carisma particolare, derivante dalla peculiare posizione ordinamentale ad essa attribuita, funzionale rispetto alla generazione del potere interpretativo. Echeggiano nella Costituzione e negli organi costituzionali chiamati a custodire e aggiornare i principi fondamentali dei sistemi politici quelle immagini di "sacralità" che erano state attribuite agli ordinamenti politici ed alle loro incarnazioni in tempi premoderni [H. Vorländer, 2006, 21].

In conseguenza della secolarizzazione dell'ordine politico e temporale si è realizzato un passaggio della sacralità al diritto ed ai suoi rappresentanti [H. Vorländer, 2006, 21].

Tale processo evolutivo, i cui albori sono riconducibili alla differenziazione fra potestas regalis e auctoritas sacrata introdotta da papa Gelasio nel IV secolo dopo Cristo ed alla riscoperta dei Digesti nell'XI secolo, si concreta nella identificazione della sacralità dello Stato con la giustizia e l'osservanza delle leggi, la signoria delle leggi acquista una veste sacrale, soprattutto in seguito alle rivoluzioni borghesi:

in tal senso il potere interpretativo della giurisdizione costituzionale sembra alimentarsi di quegli elementi sacrali connessi alla santificazione del diritto. L'autorità del diritto si traduce in tal modo nell'affermazione di una particolare autorizzazione all'interpretazione ad opera della giurisdizione costituzionale [H. Vorländer, 2006, 21]. 
Il potere interpretativo della giurisdizione costituzionale si fonda pertanto, secondo la posizione dottrinale in oggetto, sull'autorità dei giudici costituzionali quali interpreti definitivi della Costituzione: il riconoscimento di tale ruolo da parte dei destinatari dell'attività interpretativa riposa soprattutto sulle prassi istituzionali che si instaurano nei rapporti fra l'organo di giustizia costituzionale e le istituzioni politiche, fra la giurisdizione costituzionale e la giurisdizione ordinaria, nonché fra la corte (o tribunale) costituzionale e la pubblica opinione. Con specifico riferimento ai rapporti fra organo di giustizia costituzionale e legislatore ed ai fini della acquisizione da parte del primo del potere interpretativo, Vorländer distingue infine tre fasi. Tali fasi devono identificarsi con la fase di instaurazione (Etablierungsphase), che tende a coincidere con l'istituzione degli organi di giustizia costituzionale e nella quale può rivelarsi problematico il rapporto con i padri costituenti ancora in vita: la giurisdizione raggiunge una piena autonomizzazione dai medesimi solo in seguito alla loro morte biologica; con la fase conflittuale, in cui la giurisdizione costituzionale tenta di affermare la propria autorità nell'ambito dei conflitti che la oppongono agli altri poteri; infine con la fase di consolidamento, in cui il potere interpretativo non è più messo in discussione e l'organo di giustizia costituzionale tende a rafforzare la propria posizione istituzionale all'interno del sistema politico complessivamente considerato [H. Vorländer, 2006, 22-24].

\subsection{Nella dottrina nordamericana}

Con la premessa che sulle teorie elaborate dalla dottrina nordamericana in materia di legittimazione della giustizia costituzionale torneremo in seguito (par. 6 del presente capitolo) in sede di analisi delle principali teorie in materia di interpretazione costituzionale, giova fin d'ora anticipare alcune osservazioni ricostruttive in relazione ai modelli concepiti dalla dottrina medesima. I modelli di legittimazione della giustizia costituzionale possono infatti articolarsi in due categorie secondo la prospettiva di riferimento assunta, antidemocratica l'una, democratica l'altra. Il primo modello conosce due varianti, la originalista e la politica; il secondo modello si scinde in tre sub-modelli, il modello delle preferenze razionali, il modello orientato alla partecipazione e il modello orientato ai diritti [U. Haltern, 1998, 245 ss.].

Secondo il modello antidemocratico la counter-majoritarian difficulty è dilemma irresolubile ed insuperabile: la giustizia costituzionale è per se-avuto riguardo in particolare al potere di annullamento delle leggi- antidemocratica. Potendo i giudici costituzionali "devitalizzare" il risultato del processo legislativo, ne risulta violata la volontà della maggioranza. Non controllare le leggi è tuttavia, al contrario, per se una violazione del principio del costituzionalismo, in quanto maggioranze 
autonome possono perseguire in modo superficiale e contingente la tutela degli individui e delle minoranze. Ne deriva pertanto la seguente alternativa: la democrazia è desiderabile ove compatibile con i principi del costituzionalismo giudiziale (constitutionalism trumps democracy) ovvero il costituzionalismo viene in rilievo ove compatibile con la democrazia (democracy trumps constitutionalism).

L'unica via di uscita che permette di sfuggire al dilemma nascente dal rapporto di tensione che si viene ad instaurare tra democrazia e costituzionalismo consiste -secondo il metodo originalista di interpretazione della Costituzione- nell'evitare il più frequentemente possibile la counter-majoritarian difficulty. Sotto il profilo giurisprudenziale, ciò significa che il giudice deve dichiarare invalide le leggi nella minima misura possibile e solo ove contrastino con una specifica norma costituzionale. Viceversa, l'annullamento giurisdizionale si rivela illegittimo quando la norma legislativa oggetto di controllo non contrasta palesemente con una specifica norma costituzionale, risultandone altrimenti minato il processo democratico fondato sul principio di maggioranza.

Il rappresentante più autorevole di tale corrente è R. Bork, che considera quale valore costituzionale prioritario il regime democratico, in seno al quale il potere di annullamento attribuito agli organi di giustizia costituzionale rappresenta un corpo estraneo [R. Bork, 1979, 3; 1986, 639; 1990, passim]. Tale regime oscilla fra due tipi di tirannia, della maggioranza e della minoranza. La prima si manifesta quando le leggi penetrano all'interno di sfere riservate alle libertà individuali; la seconda si produce quando la maggioranza è ostacolata ad esercitare le proprie prerogative in modo legittimo. Ė compito della giurisdizione costituzionale, secondo Bork, individuare le sfere interessate dall'esercizio del potere da ritenersi sottratte alla disponibilità del regime democratico. Tale percorso interpretativo non deve fondarsi su argomentazioni di matrice giusnaturalistica, morale ovvero basate sulla teoria dei valori, né implicare l'interpretazione dello spirito o dell'idea della Costituzione: l'Autore individua tre seduzioni (temptations) cui i giudici sono esposti. La prima seduzione consiste nel prendere alla lettera la Costituzione quando impiega termini generali ed astratti: le espressioni astratte devono ricondursi e ridursi alle aspettative ed agli auspici di coloro che hanno scritto la Costituzione; ove ciò non sia possibile, perché ad esempio tali concezioni non sono più individuabili, la norma costituzionale relativa deve essere letta come una "macchia d'inchiostro", vale a dire come se non esistesse. La seconda tentazione consiste nel concepire norme costituzionali esplicite quale espressione di principi generali ed astratti da cui possano derivarsi nuove norme (ciò che ad avviso del costituzionalista statunitense si sarebbe verificato nel famoso caso Griswold vs. Connecticut del 1965). La terza tentazione consiste infine nel derivare principi astratti da pronunce costituzionali adottate in passato. 
I giudici costituzionali devono pertanto attenersi strettamente al testo, alla storia ed alle rispettive interrelazioni, nonché riferirsi alla struttura del regime politico (in particolare alla separazione dei poteri), e non possono creare nuovi diritti. Essi devono accettare ogni decisione valoriale del legislatore nella misura in cui non contrasti in modo inequivocabile con una decisione chiaramente formulata e adottata in sede di approvazione della Costituzione [cfr. in tal senso anche le posizioni di C. Wolfe, 1994; W. Rehnquist, 1976; A. Scalia, 1997].

La variante politica condivide con gli originalisti l'idea dell'incompatibilità del potere di annullamento della giurisdizione costituzionale con la democrazia e della insuperabilità della counter-majoritarian difficulty. M. Tushnet ritiene, in particolare, che i metodi dell'interpretazione costituzionale debbano conformarsi a due criteri fondamentali [M. Tushnet, 1984, 77 ss.]. Da una parte, in linea di principio essa può autorizzare in alcuni casi l'esercizio del potere di annullamento degli esiti del processo legislativo da parte dell'organo di giustizia costituzionale. Dall'altra, non può comportare l'attribuzione ai giudici della licenza di annullare leggi che ritengano il semplice prodotto di una politica sociale sbagliata. Il metodo interpretativo impiegato, in altri termini, deve prevenire il rischio che le opzioni politiche dei giudici si ritrovino nella Costituzione quali norme cogenti.

Anche per E. Chemerinsky, altro autorevole esponente della corrente politi$\mathrm{ca}$, in sede di individuazione della natura della giurisdizione costituzionale non si danno margini di conciliazione fra i due opposti estremi della abolizione di ogni controllo giurisdizionale e della sottoposizione di ogni decisione adottata in seno ad una democrazia al controllo di istituzioni ed individui politicamente responsabili in base ad un procedimento elettorale: "no theory can reconcile judicial review with majority rule (...) All judicial review in antimajoritarian, so that it is hypocritical and disingenious to single out any particular method and criticize it for being antidemocratic" [E. Chemerinsky, 1987, 11].

Analogamente agli originalisti, anche gli aderenti alla variante politica del modello antidemocratico respingono il concetto dei giudici costituzionali collocati in una posizione distinta che consentirebbe loro di comprendere in modo privilegiato lo spirito della Costituzione e rigettano la concezione che tende a differenziare le pronunce degli organi giurisdizionali dalle decisioni degli altri organi statali. Contrariamente agli interpretativisti, non ritengono tuttavia di dovere individuare un obbligo del giudice di attenersi fedelmente al testo e di rendere l'interpretazione storica il nucleo centrale di ogni interpretazione costituzionale, sostenendo anzi espressamente il judicial activism e denotando in tal senso l'intento di combinare il riparto istituzionale di ruoli tra potere legislativo e potere giudiziario da una parte e promozione del dinamismo giudiziale dall'altra. 
Secondo i rappresentanti di tale corrente, il più volte menzionato dilemma tra democrazia e costituzionalismo è suscettibile di soluzione solo ove vengano nominati giudici le cui idee collimino nei punti essenziali con quelle della maggioranza, garantendo che i loro valori personali, le loro visioni filosofiche e le loro posizioni su determinate fattispecie giuridicamente rilevanti (ad esempio in materia di aborto) siano condivise o quanto meno accettate dalla maggioranza medesima.

Gli Autori che si ispirano al modello democratico ritengono invece che la giurisdizione costituzionale favorisca e promuova il principio maggioritario e di conseguenza la democrazia. Il tentativo di conciliare democrazia e costituzionalismo (estrinsecatesi nel potere di annullamento delle leggi incostituzionali esercitato dagli organi di giustizia costituzionale) viene condotto secondo tre percorsi argomentativi diversi, il secondo ed il terzo dei quali (il modello orientato alla partecipazione ed il modello orientato ai diritti) diverranno oggetto di analisi nell'ambito del par. 6 del presente capitolo.

$\mathrm{Al}$ modello delle preferenze razionali possono ricondursi Autori quali A. Bickel e B. Ackerman.

Bickel, che ha coniato il concetto di counter-majoritarian difficulty, improntando decenni di teoria costituzionale nordamericana [A. Bickel, 1962], ha inteso individuare una soluzione di tale difficulty concependo la giustizia costituzionale quale istituzione rappresentativa. All'origine del pensiero dell'Autore si pone la distinzione fra convenienza nella direzione degli interessi della maggioranza (expediency) ed i suoi valori (principles): è compito della giurisdizione costituzionale tutelare i secondi contro i tentativi di aggressione dei primi; a differenza degli altri organi statali, il potere giudiziario viene configurato quale "pronouncer and guardian of values". In sede di esercizio di tale funzione di guardiana dei valori della comunità la giurisdizione costituzionale agisce quale istituzione rappresentativa (della maggioranza): dovendo tuttavia sempre ricondursi la legittimazione politica al consenso della maggioranza, alla giurisdizione spetterebbe tutelare $\mathrm{i}$ principles contro le expediencies solo ove un principle abbia ricevuto sostegno e condivisione da parte della maggioranza per un significativo periodo di tempo. La giurisdizione costituzionale è chiamata in tal senso a mediare fra le preferenze della maggioranza contingenti e di corte vedute ed i principi che la maggioranza medesima sosterrebbe a lunga scadenza.

Le tesi di B. Ackerman adottano quale premessa il rilievo relativo alla natura dualista della democrazia nordamericana [B. Ackerman, 1993, 1998]. In seno a quest' ultima sono rinvenibili secondo l'Autore due livelli di adozione delle decisioni politiche democratiche cui corrispondono gradi diversi di legittimità. Da un lato vi sono le decisioni realmente attribuibili al popolo, unico reale creatore di "diritto superiore" (higher law-making); dall'altro si trovano le decisioni adottate dai rappre- 
sentanti del popolo, che parimenti si traducono in "diritto", ma di diversa qualità e con un differente grado di legittimazione. Infatti, mentre le decisioni politiche ordinarie hanno luogo quotidianamente, le decisioni politiche riconducibili in via immediata al popolo si verificano in momenti eccezionali e ricorrendo speciali condizioni di mobilitazione collettiva: i momenti costituzionali. Le circostanze straordinarie che devono convergere affinché si produca uno di tali momenti sono tre: una proposta politica lanciata da un partito o gruppo di interessi riesce a coagulare un vasto consenso e a mobilitare una notevole quantità di cittadini, che identificano in tale iniziativa un progetto di peso specifico qualitativamente maggiore rispetto alle iniziative politiche ordinarie; coloro che si oppongono a tale proposta devono avere l'occasione e l'opportunità di organizzarsi e di prospettare punti di vista alternativi; la proposta deve ottenere l'adesione della maggioranza dei cittadini in seguito ad ampio ed approfondito dibattito da svolgersi nel generale foro dell'higher law-making.

Le decisioni adottate dai rappresentanti del popolo non richiedono tale mobilitazione di risorse collettive: la loro legittimità deriva dall'osservanza delle regole procedurali, che le rendano rappresentative. Non è consentito ai rappresentanti rimpiazzare con le proprie decisioni quelle adottate dal popolo e che si configurano come diritto superiore.

Momenti costituzionali sono stati secondo Ackerman la fase costituente, la fase che ha condotto alla approvazione degli emendamenti posteriori alla guerra civile e il movimento riformatore connesso al New Deal.

In seno alla democrazia dualista di Ackerman i giudici e la Corte suprema hanno la funzione di preservare l'higher law di fronte alla legislazione ordinaria: non si tratterebbe dunque, stricto sensu, di un controllo di costituzionalità, ma della salvaguardia dell'autorità delle decisioni di politica costituzionale adottate dal popolo e della tutela della linea di confine che separa i due alvei (tracks) democratici, lo straordinario e l'ordinario.

\subsection{Nella dottrina francese}

Con riferimento ai fattori capaci di accreditare o rinforzare la legittimità del controllo giurisdizionale delle leggi, L. Favoreu indica sei elementi funzionali: la storia, la forma di governo, la tecnica giurisdizionale, la Costituzione (rectius, il testo costituzionale), l'organo di controllo, la struttura dello Stato (L. Favoreu, 1986, 45 ss.].

Ricondurre la legittimità della giustizia costituzionale alla storia significa evocare le esperienze ed il retaggio storico di Paesi che hanno sperimentato periodi di dittatura, in seno ai quali è apparso assolutamente necessario istituire un controllo 
giurisdizionale delle leggi al fine di sottrarre alle maggioranze l'esclusivo dominio in materia di diritti fondamentali: gli esempi addotti dal costituzionalista francese sono quelli di Grecia, Portogallo e Spagna, che hanno sperimentato le proprie transizioni costituzionali alla metà degli anni settanta del XX secolo, per poi avviarsi su un sicuro cammino di consolidamento democratico. Possono aggiungersi i casi dei Paesi dell'Europa centro-orientale e dell'area ex-sovietica, che-come si è osservato in seno al par. 3.9 del presente capitolo- in massa hanno optato, dopo il 1989, per la creazione di sistemi di giustizia costituzionale di tipo accentrato.

La legittimità del controllo giurisdizionale delle leggi si realizza ulteriormente in funzione delle modalità di funzionamento della forma di governo. Su tale versante, l'Autore constata che i Paesi dell'Europa occidentale dotati di un sistema di controllo di costituzionalità delle leggi sono caratterizzati da una forma di governo parlamentare (Italia, Germania, Spagna, Belgio) o semipresidenziale (Austria, Portogallo, Francia). In seno alle esperienze menzionate (ad eccezione dell'Italia) vi è una maggioranza parlamentare e governativa stabile, forte e coesa, fronteggiata dall'opposizione. Quest'ultima ha bisogno di un contrappeso -la giustizia costituzionale- che la coadiuvi a fare fronte e a resistere alla maggioranza: è la logica istituzionale che porta ad erigere o sviluppare un' istituzione per reazione contro il potere eccessivo di altra istituzione [L. Favoreu, 1986, 47]. In Italia, è la debolezza e fragilità del Parlamento che implica un intervento della Corte costituzionale capace di surrogare l'assenza di scelte decisive da parte del legislatore.

La legittimità della giurisdizione costituzionale dipende inoltre dalla tecnica giurisdizionale impiegata, laddove la tecnica americana del controllo diffuso di costituzionalità appare all'Autore senza dubbio meno idonea ad assicurare la legittimità medesima rispetto alla tecnica europea del controllo accentrato (già M. Cappelletti aveva indicato varie ragioni in tale direzione). Più opinabile appare il problema relativo alla natura preventiva o successiva del controllo. Tuttavia, avuto riguardo al controllo a priori, in particolare, osserva in primo luogo Favoreu che tale tipo di controllo, lungi dal costituire un'anomalia francese, è contemplato anche in seno alle esperienze di Austria, Germania, Portogallo e Spagna; al rilievo relativo all'accentuato carattere politico normalmente annesso al controllo in via preventiva possono muoversi ad avviso dell'Autore le obiezioni secondo cui l'intervento di organi costituzionali nell'alveo del processo legislativo è previsto anche in altri ordinamenti (si pensi al rinvio presidenziale delle leggi contemplato in Germania e Italia) ed il ricorso delle minoranze parlamentari o di altri soggetti costituzionali all'organo di giustizia costituzionale è tipico di tutti i Paesi in cui si accoglie il controllo astratto di costituzionalità delle norme. Il controllo a posteriori, d'altra parte, non si sottrae al rilievo relativo alla invasività della dichiarazione di illegittimità costituzionale rispetto alle prerogative ed ai poteri del legislatore ove 
si traduca nella demolizione dell'intero edificio giuridico creato da quest' ultimo, nonché all'osservazione tendente a sottolineare la natura acrobatica dei rimedi cui i singoli ordinamenti sono costretti a fare ricorso al fine di conciliare gli effetti derivanti dall'annullamento di una o più norme con la certezza e sicurezza dei rapporti giuridici.

La legittimità della giustizia costituzionale trova la propria fonte, inoltre, nella stessa natura del testo costituzionale, il cui carattere sfumato, impreciso od evanescente e la cui origine storica eventualmente assai risalente nel tempo esaltano -e non svalutano- la funzione adeguatrice del giudice costituzionale.

L'organizzazione ed il funzionamento dell'organo di giustizia costituzionale si configurano quali ulteriori fattori di condizionamento della legittimità del medesimo. Su tale versante, a fronte della critica relativa alla natura non democratica e non rappresentativa delle corti o tribunali costituzionali, Favoreu pone in evidenza la caratteristica comune alla maggioranza delle corti stesse che le vede composte da membri designati in prevalente misura da organi elettivi dotati di piena democraticità e rappresentatività: la politicizzazione della designazione e della composizione delle giurisdizioni costituzionali, lungi dall'essere una tara, si rivela un elemento necessario di tali sistemi.

La legittimità della giustizia costituzionale in funzione della struttura dello Statoordinamento, infine, è resa evidente dalla necessità del controllo di costituzionalità delle leggi negli Stati federali e regionali, ovvero in seno alle esperienze fondate su un delicato equilibrio fra comunità etniche e linguistiche (Belgio).

Il controllo di costituzionalità può contribuire, a diverso titolo, a migliorare il funzionamento dei regimi democratici grazie agli effetti benefici che è in grado di produrre in seno alla sfera giuridica e politica: in tale direzione Favoreu individua sei funzioni fondamentali che la giustizia costituzionale è chiamata ad esplicare e che, nel loro complesso, denotano l'attitudine ad incrementare la legittimità della stessa: la funzione di pacificazione della vita politica, la funzione di regolazione e autenticazione dei mutamenti politici e dell'alternanza, la funzione di rafforzamento della coesione della società politica, la funzione di diffusione $\mathrm{e}$ radicamento dei diritti fondamentali, la funzione di protezione dei diritti fondamentali, la funzione di adeguamento e aggiornamento della Costituzione [L. Favoreu, 1986, 62-66].

Una configurazione della giurisdizione costituzionale di natura dinamica in quanto modello adattabile alla mutevole realtà della democrazia nella sua fase attuale, presentandosi come democrazia continua, è stata elaborata in seno alla dottrina francese, in particolare, da D. Rousseau e presuppone la affermazione di tre nuove forme di rappresentazione delle opinioni nella competizione politica, la forma dei sondaggi, la forma mediatica, che si realizza attraverso i mezzi di comu- 
nicazione, la forma costituzionale, che si attua mediante i tribunali costituzionali o, nel caso della Francia, il Consiglio costituzionale [D. Rousseau, 1995, 7 ss.; 1998, 139 ss. e, più recentemente, 2006].

Diversamente dalla scuola positivista e da quella del diritto naturale, che fondano la legittimità della giustizia costituzionale su una definizione a priori della democrazia, l'Autore propone un'altra ipotesi, che viene formulata nei seguenti termini: il controllo di costituzionalità delle leggi è legittimo perché produce una definizione della democrazia che lo legittima [D. Rousseau, 2006, 2]. Obiettivo di individuazione da parte di $\mathrm{D}$. Rousseau non è il rapporto che intercorre fra verità democratica e meccanismo di controllo di costituzionalità, ma la verità democratica che tale meccanismo rende possibile, di conseguenza legittimandolo.

Oggi la giustizia costituzionale produce un nuovo linguaggio, "una nuova rappresentazione della democrazia che fonda la legittimità della ricomposizione dello scenario istituzionale sul principio della preminenza della Costituzione e di conseguenza del giudice costituzionale" [D. Rousseau, 2006, 3]. Tale nuova configurazione della democrazia, altrove denominata dall'Autore "democrazia continua" [D. Rousseau, 1995, 9 ss.], "legittima un giudice costituzionale che, attraverso la sua attività giurisdizionale, si comporta come il costruttore dell'autonomia dello spazio pubblico e il costruttore del farsi norma della parola del sovrano" [D. Rousseau, 2006, 4].

La prima delle prospettive delineate è dominata da un epocale capovolgimento di scenario, caratterizzato dal passaggio dalla Costituzione-separazione dei poteri alla Costituzione-garanzia dei diritti e dal connesso sviluppo della giurisprudenza costituzionale.

Questa nuova concezione della costituzione, risultato della giurisprudenza costituzionale, ha una incidenza diretta sulla rappresentazione della relazione governati-governanti. La carta, in effetti, definisce progressivamente uno schermo, o più precisamente, uno spazio che assicura simbolicamente e praticamente -attraverso la censura eventuale della leggel'autonomia dei rappresentati rispetto ai rappresentanti. (...) La giurisprudenza produce così una configurazione nuova di distanziamento dei governati e dei governanti, fondando i diritti dei primi come un corpo separato dai diritti dei secondi: la carta giurisprudenziale dei diritti e delle libertà costituzionali rappresenta lo spazio dei governati, la legge, invece, lo spazio dei governanti [D. Rousseau, 2006, 4-5].

Lo spazio costruito tra la società civile e la società politica è occupato dunque dalla giurisdizione costituzionale; il principale effetto prodotto dalla distinzione governanti-governati nel senso dianzi descritto consiste nella rappresentazione della rappresentazione:

giudicando delle leggi rispetto alla volontà costituzionale del popolo, il Consiglio [costituzionale, n.d.r.] rende evidente che i rappresentanti non sono i sovrani ma sono soltanto 
$i$ delegati del potere sovrano; ogni decisione rappresenta la stessa scena, quella in cui $i$ governanti avendo parlato e deciso in nome del popolo sono, improvvisamente, ricondotti alla loro condizione di semplici delegati e messi a confronto, per esservi finalmente sottomessi, alla fonte della loro delega. E questa scena produce, d'un colpo, l'immagine del popolo come sovrano poiché è rispetto ai suoi diritti, alla sua volontà, che sono giudicate le azioni normative dei suoi delegati [D. Rousseau, 2006, 6].

Se l'affermazione della superiorità del popolo rispetto ai suoi rappresentanti si realizza attraverso la mediazione dell'organo di giustizia costituzionale, lo specchio che riflette la sua immagine di popolo sovrano è -nella ricostruzione di D. Rousseau-carta giurisprudenziale dei diritti e delle libertà che il giudice costituzionale presenta al popolo per renderlo consapevole della propria sovranità ed ai rappresentanti per renderli consapevoli della loro subordinazione al sovrano.

In seno alla seconda delle prospettive delineate, la democrazia continua sembra caratterizzata da una (apparente) contraddizione, individuabile nel mezzo -l'organo di giustizia costituzionale (di natura non elettiva) - che realizza la mediata -e non diretta- superiorità del popolo rispetto ai suoi rappresentanti. Tale antinomia si supera, secondo D. Rousseau, considerando che, nel caso del Consiglio costituzionale,

non si tratta, in effetti, di un ulteriore rappresentante del popolo sovrano, al fianco del Parlamento e dell'Esecutivo; è l'istituzione che (ra)ppresenta al popolo e ai suoi delegati la sovranità popolare, cos̀̀ come appare nella costituzione. La differenza è importante. Quando il Consiglio censura una legge, non lo fa perché i rappresentanti hanno travisato la volontà dei cittadini che li hanno eletti; non lo fa neanche perché conosce, e dunque rappresenta meglio degli eletti, la volontà del popolo che si espressa al di fuori di quelle elezioni; il Consiglio censura (...) il testo nel quale la sovranità del popolo si esprime e che impedisce loro di assumere quelle decisioni. In altri termini, il Consiglio non rappresenta il popolo sovrano, egli rappresenta quello nel quale, e mediante il quale, il popolo si immagina e si riconosce sovrano [D. Rousseau, 2006, 10].

Attraverso la propria opera interpretativa, l'organo di giustizia costituzionale conferisce normatività alla Costituzione entrando in un gioco di rapporti di forza con altre istituzioni, di natura diversa, contribuendo nell'ambito della democrazia continua -nella sua veste di imprenditore privilegiato fra gli imprenditori legislativi (entrepreneurs législatifs) che nel loro complesso partecipano alla enunciazione della norma- alla delineazione di un regime di affermazione concorrenziale delle norme: da una parte, infatti, la formazione della legge appare oggi il frutto del lavoro di tre istituzioni concorrenti (Parlamento, Governo, lo steso organo di giustizia costituzionale), dall'altra l'interpretazione del Consiglio costituzionale non è mai frutto di scelte totalmente libere ed arbitrarie, ma la conseguenza di una serie di vincoli, "di un gioco che mette in concorrenza diversi attori": i parlamentari, i professori 
di diritto, le organizzazioni di categoria interessate, la stampa specializzata o meno, la stessa giurisprudenza costituzionale:

il Consiglio non è dunque libero dalle sue interpretazioni. Deve integrare o, almeno, tenere conto delle interpretazioni concorrenti. Da ciò dipende la sua legittimità di interprete, poiché, al di là del suo fondamento costituzionale, essa riposa anche sul riconoscimento del valore della sua giurisprudenza da parte della comunità giuridica e politica. Ora questo riconoscimento, per costruirsi, presuppone che fra il Consiglio e i suoi interlocutori si stabilisca un rapporto nel quale i secondi, malgrado la posizione istituzionale del primo, possano ritrovarsi, in tutto o in parte, nella motivazione delle decisioni; in assenza di ciò, il Consiglio rischierà di provocare l'ostilità delle istituzioni delle quali ha bisogno per garantire la sua legittimità [D. Rousseau, 2006, 12].

Concorrenziale e deliberativo, il nuovo regime di enunciazione delle norme indica un fondamentale mutamento di contesto rispetto a quello della democrazia rappresentativa, un contesto in seno al quale il giudice costituzionale svolge un ruolo privilegiato. La conclusione che può condividersi è che

la necessità e legittimità di una giustizia costituzionale ai nostri tempi vengono date all'importante e indispensabile ruolo che svolge nell'ultimo tipo di democrazia: la arricchisce con la protezione dei diritti degli individui e delle minoranze, apre nuovi spazi di partecipazione individuali e settoriali, potenzia il pluralismo e la concorrenza nei processi pubblici, rappresenta in modo nuovo la pubblica opinione (...), perfeziona la formazione della volontà generale, che non si configura più come volontà di una semplice maggioranza parlamentare, ed amplia il campo della deliberazione nella produzione delle norme, oltrepassando parimenti i limiti imposti dalla democrazia rappresentativa pura [J. Acosta Sanchez, 1998, 369].

\subsection{Nella dottrina iberoamericana}

Accanto a ragioni giustificative di natura tradizionale, vengono addotte da N. P. Sagüés quattro argomentazioni fondamentali ai fini della individuazione dei fattori di legittimazione della giustizia costituzionale [N. P. Sagüés, 2002, 51 s.].

Il primo di tali fattori viene identificato dal costituzionalista argentino con il procedimento concreto di selezione e designazione dei giudici costituzionali (ad esempio mediante le assemblee rappresentative) che, in quanto programmato da una Costituzione democratica, gode di una significativa quota di legittimazione democratica. Si tratta di legittimazione di secondo (o terzo grado), ma pur sempre derivante dal sistema costituzionale che consente alla legittimazione medesima di trasmettersi all'organo di giustizia costituzionale attraverso i meccanismi a tal fine previsti.

Il secondo fattore di legittimazione individuato dall'Autore concerne la formazione ed il patrimonio culturale dei giudici costituzionali. Ricordando che la legitti- 
mazione democratica non deriva solo dalle elezioni, ma anche da altre procedure democratiche di selezione, Sagüés osserva come una versione più raffinata e matura della democrazia contemporanea esige, ad esempio e con particolare riferimento alla giurisdizione costituzionale, l'implementazioine di discipline di reclutamento dei giudici capaci di soddisfare i principi democratici di eguaglianza di opportunità e di selezione fondata su criteri di idoneità e la rinuncia a forme discrezionali di nomina capaci di ingenerare fenomeni di parzialità e favoritismo: in tal senso, la nomina dei giudici preceduta da fasi preventive di formazione e specializzazione, concorsi e prove attitudinali ecc. è in grado di conferire ai medesimi un'alta dose di legittimità democratica [N. P. Sagüés, 2002, 51].

Il terzo fattore di legittimazione indicato dall'Autore coincide con la legittimità di esercizio della giurisdizione costituzionale. Si ritiene infatti che la legittimazione democratica degli organi di giustizia cositutzionale non possa essere ricondotta esclusivamente ad una problematica di origine democratica, ma anche (ed in larga misura) alle modalità di comportamento dei suoi quadri:

tale "condotta democratica" obbliga i giudici che controllano la supremazia costituzionale ad emanare le proprie pronunce in quanto custodi della costituzione democratica che devono tutelare di fronte alle violazioni che possano commettere gli altri organi dello Stato (o i tribunali inferiori), e con senso del bene comune, che non sempre coincide con la volontà della maggioranza contingente. Detto in altre parole, e come ha indicato la Corte Suprema dello Iowa in "Hunter v. Colfax", la Costituzione protegge il popolo dagli eccessi dei governanti e dagli eccessi dello stesso popolo, cosa che conviene sempre tenere presente [N. P. Sagüés, 2002, 51-52].

La quarta ragione argomentativa configurata da Sagüés si fonda sulla compatibilità tra democrazia ed efficacia del sistema istituzionale. L'Autore ricorda infatti come il regime democratico non debba manifestarsi ncessariamente in forme pure o assolute quando le medesime sono in grado di pregiudicare la funzionalità del sistema politico. Gli esempi che l'Autore offre riguardano, fra gli altri, la carriera militare, diplomatica o giudiziaria: la simbiosi necessaria che deve instaurarsi in tali casi fra democrazia ed efficacia è requisito fondamentale ai fini della stabilità e persistenza dell'assetto democratico [N. P. Sagüés, 2002, 52].

Il tentativo di individuare le ragioni giustificative della legittimità della giustizia costituzionale al di fuori -quanto meno parzialmente- del circuito democraticorappresentativo strettamente inteso, anche mediante il riferimento a valori e principi metademocratici, è altresì rinvenibile nell'opera di A. R. Brewer-Carias [A. R. Brewer-Carias, 1996, VI, 65 ss.]. Il dibattito sulla legittimità/illegittimità del potere conferito ad organi statali non responsabili di fronte al popolo di controllare gli atti di altri organi invece responsabili, e -conseguenzialmente- sul carattere democratico o non democratico del controllo giurisdizionale 
può considerarsi non solo come interminabile e astratto, ma anche bizantino, in particolare in quanto si fonda su un supposto problema di legittimità astratta del controllo giurisdizionale, che potrebbe risolversi solo in forma astratta. Il problema del controllo giurisdizionale della costituzionalità o dei poteri attribuiti ai giudici od organi costituzionali di natura giurisdizionale per vigilare sulla costituzionalità delle leggi, non può essere trattato o discusso sotto il profilo della legittimità o illegittimità, partendo dal principio che la democrazia si basa solo sulla rappresentatività. La democrazia va al di là della cornice della sola rappresentatività, poiché si tratta piuttosto di un modo politico di vita, in cui hanno primazia le libertà individuali ed i diritti fondamentali degli esseri umani. Ciò è tanto vero che si può affermare che un sistema di controllo giurisdizionale effettivo della costituzionalità delle leggi non è praticabile in regimi non democratici, soprattutto perché in tali sistemi non può esistere una reale indipendenza dei giudici, essendo assolutamente chiaro che non si può esercitare un controllo giurisdizionale effettivo in sistemi in cui non si garantisce l'autonomia e l'indipendenza del potere giudiziario [A. R. Brewer-Carias, 1996, VI, 66].

Democratico e legittimo è dunque il potere riconosciuto ai giudici o a determinati organi costituzionali affinché vigilino sulle violazioni dei diritti fondamentali in quanto il potere medesimo è immanente e connaturato ad ogni regime rappresentativo e democratico e finalizzato al rafforzamento delle libertà del cittadino.

La legittimità democratica del controllo giurisdizionale della costituzionalità delle leggi deriva dunque dal rapporto di immedesimazione che intercorre fra la tutela dei diritti fondamentali ed il sistema democratico (prima ragione giustificativa della legittimazione) e si fonda ulteriormente sulle modalità di distribuzione dei poteri adottato dalla Costituzione, sia a livello verticale che a livello orizzontale, sia in sede di articolazione dello Stato-ordinamento che di strutturazione dello Statoapparato (seconda ragione giustificativa della legittimazione). Tuttavia-osserva il costituzionalista venezuelano-se il problema della legittimazione non si è mai posto sul versante della distribuzione verticale del potere fra i diversi livelli di governo in seno a forme di Stato federali, regionali o comunque improntate al decentramento, risultando intrinseca al federalismo l'imposizione di un certo grado di supremazia della Costituzione e delle leggi federali sulla normativa degli Stati membri, esso è invece emerso sul versante della separazione (orizzontale) dei poteri, principio ritenuto oggetto di erosione in conseguenza della limitazione della supremazia del Parlamento ad opera del controllo di costituzionalità. Anche su tale versante, tuttavia, non viene meno il criterio giustificativo dianzi prospettato: la separazione dei poteri ha implicato la previsione di un meccanismo indipendente per garantire il contenuto organico della Costituzione: l'indefettibilità della giurisdizione costituzionale in quanto strumento operante nel sistema costituzionale di pesi e contrappesi deriva dalla necessità di conservare l'equilibrio su cui si regge la Costituzione medesima. 
Il controllo di costituzionalità (e l'organo che lo esercita) non appare, nella ricostruzione di Brewer-Carias, un elemento spurio rispetto alla separazione dei poteri: l'apparente collisione fra la necessità di instaurare un controllo giurisdizionale della Costituzione ed il principio della separazione dei poteri è antinomia che si risolve aggiornando la concezione del principio medesimo, considerandola inclusiva di organo e procedura ulteriori rispetto alla connotazione tradizionale.

La legittimità del controllo giurisdizionale di costituzionalità procedente dalla legittimità dello Stato costituzionale che determina la forza normativa della Costituzione e la necessità della sua protezione contro l'azione dei poteri costituiti che intendano violarla è sillogismo centrale nel pensiero di H. Nogueira [H. Nogueira, 2004,13 ss.]. La pretesa invasione da parte della giurisdizione costituzionale della sfera propria del potere legislativo è critica che trascura l'esistenza, ad avviso del costituzionalista cileno, di una divisione più profonda fra il potere costituente ed i poteri costituiti, in seno alla quale la giurisdizione costituzionale si sviluppa per proteggere la Costituzione contro i "marosi” (embates) suscitati dagli organi costituzionali "costituiti":

la giurisdizione costituzionale garantisce la forza normativa della Costituzione, che rende possibile concepirla come norma giuridica vincolante e non solo come una proclamazione politico filosofica (...) In tal modo, la giurisdizione costituzionale si legittima per il passaggio dallo Stato legale allo Stato costituzionale di diritto ed il riconoscimento della Costituzione come norma giuridica superiore e obbligatoria per i poteri costituiti [H. Nogueira, 2004, 13-14].

Alla critica che sottolinea la carenza di legittimazione della giurisdizione costituzionale rispetto alla rappresentanza popolare e la irresponsabilità della prima nei confronti del corpo elettorale, l'Autore muove cinque obiezioni fondamentali.

La legittimità democratica della giurisdizione costituzionale viene fatta discendere, in primo luogo, dalla stessa decisione e legittimità del potere costituente che stabilisce la Costituzione, potere che conferisce legittimità agli organi costituiti e ne determina la forma di integrazione. A ciò si aggiunge il rilievo secondo il quale nella maggior parte dei casi i membri degli organi di giustizia costituzionale sono eletti o designati da organi politici (Parlamento e Governo), fattore che fa sì che la giurisdizione costituzionale sia dotata di adeguata rappresentatività [H. Nogueira, 2004, 14].

Si ricorda, in secondo luogo, come le decisioni delle maggioranze presenti nell'ambito degli organi politici non sempre rappresentino la volontà del corpo politico della società ovvero rispettino i diritti fondamentali delle persone e dei gruppi più deboli in seno alla medesima, in quanto spesso costituiscono maggioranze "artificiali", prodotto dei sistemi elettorali, che non fungono da specchio fedele delle articolazioni della società: la giurisdizione costituzionale si propone in tal senso quale istituzione destinata a proteggere $i$ diritti fondamentali contro 
eventuali abusi od arbitri degli organi politici, a conservare la distribuzione delle potestà e competenze determinata dalla Costituzione, razionalizzando e inquadrando giuridicamente l'azione degli attori politici, risolvendo i conflitti, rafforzando il funzionamento dello Stato costituzionale democratico, proteggendo i diritti dei singoli e dei gruppi, incrementando la qualità della democrazia ed una adeguata governabilità [H. Nogueira, 2004, 15].

La terza ragione giustificativa della legittimità della giurisdizione costituzionale è data secondo Nogueira da due fattori: da una parte dalla regola generale secondo la quale agli organi di giustizia costituzionale è preclusa normalmente la possibilità di procedere ex officio (le pronunce dei medesimi dovendo essere sollecitate da istanze o ricorsi di terzi, organi politici, minoranze parlamentari, organi di tutela dei diritti fondamentali o persone che ritengano violate le proprie situazioni giuridiche soggettive), dall'altra, per il fatto che le decisioni di tali organi devono trovare una giustificazione giuridica e razionale, essere dotate di fondamento, congruenti e fondate sulle fonti del diritto costituzionale vigente, ciò che rende possibile un controllo della comunità scientifica (in particolare, giuridica) e della società nel suo complesso sull'operato delle corti (o tribunali) costituzionali [H. Nogueira, 2004, 15-16].

Una prova addizionale della legittimità della giurisdizione costituzionale è fornita secondo l'Autore dalla crescente accettazione generalizzata della medesima da parte dei costituenti democratici degli Stati costituzionali che, agli albori del XXI secolo, nei diversi continenti realizzano processi di transizione costituzionale ed esperiscono tentativi di consolidamento democratico: in tali contesti la giustizia costituzionale viene concepita quale pilastro ed asse fondamentale attorno al quale si sviluppa e consolida la democrazia costituzionale, in quanto non meramente rappresentativa ma anche deliberativa e continua, in seno alla quale i giudici costituzionali contribuiscono ad esprimere la volontà attuale attraverso la costruzione della giurisprudenza costituzionale [H. Nogueira, 2004, 16].

Si relativizza, infine, il potere della giurisdizione costituzionale, "la cui parola non è l'ultima parola", considerando che il corpo politico della società ed il potere di revisione costituzionale, se ritengono che i giudici costituzionali hanno oltrepassato l'idea di diritto valida e vigente in seno alla società, possono sempre modificare il testo costituzionale, inducendo la giurisdizione costituzionale ad agire nella direzione da esso indicata [H. Nogueira, 2004, 16].

La ricostruzione delle funzioni, dei fini e delle ragioni giustificative della legittimazione della giustizia costituzionale improntata da maggiore sistematicità è rinvenibile nella dottrina spagnola in seno all'opera di J. Acosta Sánchez [J. Acosta Sánchez, 1998, 341 ss.].

L'Autore identifica tre fini fondamentali che la giurisdizione costituzionale è chiamata a perseguire: la depurazione dell'ordinamento giuridico da norme incostituzio- 
nali e la purificazione della legge, la garanzia dei diritti e delle libertà fondamentali, la risoluzione dei conflitti di competenza, in particolare sul versante dell'organizzazione territoriale del potere [J. Acosta Sánchez, 1998, 349]. Ai tre fini fondamentali altri se ne aggiungono di natura non scritta: la partecipazione al processo legislativo $o$ creazione di norme sul piano legislativo, la creazione di norme costituzionali, la costituzionalizzazione di tutte le branche del diritto [J. Acosta Sánchez, 1998, 354].

Le esplicazioni fornite dalla dottrina del complesso di tali funzioni vengono classificate dall'Autore in due categorie, quella delle esplicazioni esorbitanti e quella delle esplicazioni plausibili [J. Acosta Sánchez, 1998, 362 ss.].

Rientrano nella prima categoria la configurazione dell'organo di giustizia costituzionale come terza Camera, la concezione della giurisdizione costituzionale come "potere costituente continuo", "costituente secondario" e "esecutrice o commissaria del potere costituente" e la considerazione della giustizia costituzionale come "paradosso" e fondamen-talmente antidemocratica.

La prima esplicazione esorbitante della giurisdizione costituzionale risale al XIX secolo e la presenta come una terza Camera legislativa con veto assoluto:

la catalogazione della giurisdizione costituzionale quale terza Camera legislativa ha trovato i suoi motivi più fondati nella creatività, attivismo e politicizzazione di cui è stata accusata. Mediante la prima si riprova l'organo della giurisdizione costituzionale non come semplice "legislatore negativo", ma come legislatore positivo, creatore di disposizioni legislative senza legittimazione costituzionale e senza le responsabilità politiche annesse al legislatore. Se tale intromissione nel potere legislativo si produce in forma continuata o intensa si parla di attivismo. Se ha luogo seguendo flagranti criteri di opportunità e sulla base di schieramenti notoriamente di parte, si parla di politicizzazione. (...) L'esplicazione peggiorativa o accusatoria della giurisdizione costituzionale come "terza Camera legislativa" incide infine sul ruolo da essa giocato ai fini della configurazione della rappresentanza politica e della funzione dei partiti politici, rendendo più trasparente e accentuando la crisi attuale della democrazia rappresentativa [J. Acosta Sánchez, 1998, 363-364].

Altre esplicazioni "esorbitanti" presentano la giurisdizione costituzionale come potere costituente continuo, costituente secondario e commissaria del potere costituente.

La prima configurazione ha origine nell'esperienza nordamericana ed è stata coniata da W. Wilson in sede di qualificazione della Corte Suprema quale continous constitutional Convention [W. Wilson, Congressional Government, 1895. Gli sviluppi evolutivi di tale dottrina sono analiticamente affrontati in seno al par. 6 del presente capitolo]:

si tratta di una spiegazione inevitabile avuto riguardo alla struttura e storia del diritto costituzionale originario (USA), formalmente marcato dalla atrofia del potere di revisio- 
ne costituzionale (amending power), disciplinato in modo occlusivo nell'articolo $V$ della Costituzione, e reso materialmente ipertrofico dall'azione continua svolta per più di due secoli da un potente potere giudiziario attraverso il judicial review. Lidea di una giurisprudenza costituente acquista così senso nello straordinario ambito storico della Corte Suprema statunitense, ma non è trasferibile all'Europa della Costituzione normativa di questa seconda metà del secolo, né è utile al fine di penetrare la natura delle giurisdizioni costituzionali [J. Acosta Sánchez, 1998, 365].

L'espressione che presenta il giudice costituzionale come un costituente secondario è utilizzata in Francia dalla teoria realista dell'interpretazione costituzionale, che ha in M. Troper la sua figura più rappresentativa [M. Troper, 1998, in particolare 305 ss.]. Secondo tale teoria, ogni interpretazione assume una funzione creativa, traducendosi in un atto di volontà in virtù del quale l'interprete ricrea il testo, che manca di senso oggettivo prima dell'opera interpretativa. Gli organi di giustizia costituzionale, in particolare, nella veste di interpreti supremi della Costituzione, capaci di rendere un'interpretazione "autentica" in quanto investiti dalla medesima del potere di interpretarla, forniscono un'interpretazione che si incorpora in seno all'ordinamento giuridico a prescindere dal significato che assume, e non essendo le loro decisioni suscettibili di annullamento, si deve considerare che partecipino all'esercizio del potere costituente, come un costituente secondario.

La terza esplicazione della giurisdizione costituzionale dianzi accennata contempla l'organo competente come esecutore o commissario del potere costituente, definendolo quale istanza commissionata dalla Costituzione [R. Marcic, 1963, 204], come esecutore della volontà del potere costituente [A. Weber, 1986, 70] ovvero concependone la posizione giuridica come commissario del potere costituente [E. García de Enterría, 1981, 197]. Si tratta di posizioni cui sono state mosse varie critiche, che hanno trovato la loro matrice, in particolare, nella coerenza dei fondamenti della Costituzione democratica che, se correttamente intesa, impedisce

di attribuire in qualche forma potere costituente ad alcun potere costituito, cosa che è il tribunale costituzionale. Un organo di natura giurisdizionale non può avere contestualmente natura costituente e costituire formalmente e originariamente lo Stato. Se, come accade in realtà, le giurisdizioni costituzionali di tutti i tipi creano la Costituzione, non può in nessun caso trattarsi della Costituzione formale, opera del potere costituente sovrano, ma di altra cosa, norme materialmente costituzionali, diritto costituzionale giurisprudenziale [J. Acosta Sánchez, 1998, 366].

La terza esplicazione esorbitante concepisce la giurisdizione costituzionale come "paradossale" e antidemocratica.

Il paradosso del giudice costituzionale è stato configurato come un caso applicativo del paradosso generale del giudice in una società democratica: il paradosso 
consisterebbe nella necessità del controllo giurisdizionale delle leggi prodotte da assemblea formata da soggetti che -si presume- rappresentano la volontà del popolo, o quanto meno della sua maggioranza. Esiste un parallelo -si è affermato- fra la situazione del giudice ordinario, che non è mai totalmente fedele alla democrazia in quanto nella sua interpretazione della legge corre sempre il rischio di allontanarsi dalla volontà del legislatore, e quella del giudice costituzionale, che si vede obbligato ad adottare, o quanto meno ad instaurare, posizioni antimaggioritarie, secondo quanto accade in sede di tutela dei diritti fondamentali dell'individuo contro le violazioni derivanti da opzioni riconducibili alla volontà della maggioranza parlamentare [M. Rosenfeld, 1995, 104]. Si tratta di posizione dottrinale che non ha mancato di sollevare critiche dirette a porre in luce, in particolare, il fatto che

prende le mosse da una semplificazione fondata su un paradigma confuso: si colloca nella generalità del regime parlamentare, non nell'ambito specifico corrispondente, che è quello della democrazia costituzionale o dello Stato costituzionale democratico. In questultimo, il legislatore non è democratico per se, sebbene esprima la volontà della maggioranza parlamentare, ma solo nel rispetto della Costituzione, che esprime una volontà superiore alla propria, quella del popolo sovrano. Quando il giudice costituzionale annulla una legge in quanto incostituzionale non incorre pertanto in un paradosso, al contrario restaura la coerenza tra la volontà suprema del sovrano, fonte prima del diritto, e l'opera dei suoi rappresentanti [J. Acosta Sánchez, 1998, 366].

D'altra parte, la conclusione secondo cui l'interpretazione giudiziale delle leggi è inevitabilmente antidemocratica in considerazione della soggettività inerente alla medesima e della forza politica di cui soffrono le decisioni dei giudici [R. Unger, 1983, 561 ss.] non risulta applicabile alle giurisdizioni costituzionali europee, in cui il giudice costituzionale gode di piena legittimità costituzionale: la verifica della sua obiettività passa attraverso l'analisi dei metodi di interpretazione costituzionale ed in particolare delle esplicazioni positive o plausibili che della giurisdizione costituzionale sono state offerte da una molteplicità di correnti dottrinali [J. Acosta Sánchez, 1998, 367].

Rientrano nella categoria delle esplicazioni plausibili o possibiliste della giurisdizione costituzionale la già analizzata esplicazione realista di V. Crisafulli (vedi supra) che la concepisce come un'alterazione necessaria dello schema democratico-parlamentare, l'esplicazione dinamica, che la vincola ad un nuovo paradigma democratico, quello della democrazia continua (parimenti analizzata supra in sede di ricostruzione delle principali correnti dottrinali francesi), infine l'esplicazione contestuale, che contempla l'espansione ed il potenziamento delle giurisdizioni costituzionali quale risultato della dinamica dei contesti, in particolare del contesto sociale. 


\subsection{Funzioni e legittimazione della giustizia costituzionale}

Il ruolo e le funzioni degli organi di giustizia costituzionale nelle democrazie contemporanee -e correlativamente le ragioni giustificative della loro legittimazione in seno alle medesime- possono divenire oggetto di valutazione in una prospettiva parziale e settoriale (coincidente con il giudizio di costituzionalità delle leggi) ovvero in una prospettiva sistemica, tale da abbracciare le funzioni degli organi stessi nel loro complesso.

Se si adotta come parametro di riferimento la funzione consistente nel controllo della legittimità costituzionale delle leggi, ci si avvede come il ruolo svolto dagli organi di giustizia costituzionale su tale versante si concreti nella tendenziale avocazione ad una sede diversa ed ulteriore rispetto a quella politica del processo di ricognizione e svolgimento dei valori costituzionali, nel contributo fornito dal giudice costituzionale alla precisazione del contenuto delle norme costituzionali mediante un'opera ermeneutica che, secondo le diverse ipotesi, affianca, integra, corregge o finanche sostituisce le scelte effettuate dal legislatore.

Si realizza, nel modo descritto, una ponderazione complementare e sussidiaria degli interessi emergenti in seno alla società rispetto ad ogni singolo ambito materiale che, nelle ipotesi più qualificanti, si traduce in una sorta di drenaggio assiologico da parte del giudice costituzionale, che contribuisce alla individuazione dei principi-cardine e dei valori-guida cui deve necessariamente conformarsi il legislatore che intenda porre mano alla disciplina o all'adeguamento normativo di una determinata materia.

Gli organi di giustizia costituzionale, d'altra parte, non mancano di proporsi, sotto il profilo indicato, quali custodi del rispetto delle regole costituzionali su cui si è realizzato il consenso: la funzione svolta dal giudice costituzionale nell'ottica descritta, pertanto, non è unicamente finalizzata alla realizzazione del consenso, bensì anche alla preservazione, alla salvaguardia ed al consolidamento del consenso circa le regole medesime.

Assecondando, completando o censurando le scelte normative operate dal legislatore, gli organi di giustizia costituzionale esplicano inoltre, nel senso dianzi precisato, una funzione di legittimazione del contenuto degli atti legislativi, il cui vaglio e verifica da parte del giudice costituzionale si traduce in una sorta di certificazione di costituzionalità nelle ipotesi di controllo di legittimità costituzionale in via preventiva (come nell'esperienza francese) o nella determinazione e attestazione della conformità alla Costituzione della proposizione normativa forgiata dal legislatore, quindi nella legittimazione del contenuto che caratterizza la proposizione medesima. Tale determinazione, inoltre, diviene particolarmente incisiva ove alle sentenze costituzionali venga riconosciuta forza di legge.

Gli organi di giustizia costituzionale dispongono di regola di un'articolata tipologia di pronunce che consente al giudice costituzionale di modulare e variare 
l'impatto politico delle proprie sentenze, che divengono strumento utilizzato con diversa duttilità secondo il grado di ingerenza all'interno della sfera discrezionale del legislatore che il giudice medesimo intende raggiungere.

La morfologia delle pronunce costituzionali risulta in tal modo estremamente variegata ed è caratterizzata dalla presenza, in seno all'arco i cui poli estremi sono rappresentati dalle sentenze di mero accoglimento o rigetto della questione di costituzionalità, di tipi di pronunce che rendono estremamente elastici i margini di operatività che residuano a favore dei titolari del potere legislativo nella loro opera di disciplina o di riforma delle singole materie.

La disponibilità dell'ampio strumentario giurisprudenziale menzionato induce d'altra parte il giudice costituzionale a concepire in chiave parimenti duttile ed elastica il principio del self-restraint e a relativizzare largamente il principio, al primo strettamente connesso, della astensione da pronunce che concernano o lambiscano political questions unicamente di spettanza del legislatore. La tendenza seguita dagli organi di giustizia costituzionale si concreta anzi nella configurazione da parte dei medesimi di ipotesi e soluzioni alternative a favore del legislatore che intenda porre mano alla disciplina o alla riforma di singole fattispecie, anche se all'interno di un quadro determinato dal giudice costituzionale e mediante l'apposizione di limiti generalmente precisi, di regola accompagnata dal monito di provvedere nel senso auspicato o dalla minaccia di sanzionare in sede di processo costituzionale opzioni diverse eventualmente preferite dal legislatore in sede parlamentare. Dall'utilizzazione dello strumentario giurisprudenziale nel senso descritto sembra trasparire l'intento del giudice costituzionale di valorizzare il ruolo e le funzioni di moderazione, di critica e di decantazione del processo decisionale svolto dalle minoranze, dai gruppi di interesse, dai soggetti esponenziali di interessi collettivi o diffusi, che risulterebbe altrimenti privo di interlocutori fondamentali, che trovano nella giustizia costituzionale una sede di rappresentazione delle proprie istanze.

È già sulla base delle osservazioni svolte che emerge la necessità di contemplare le giurisdizioni costituzionali non solo come "custodi negativi" della Costituzione, chiamati a depurare l'ordinamento giuridico delle norme incostituzionali create dagli altri poteri dello Stato [L. Garlicki-W. Zakrzewski, 1985, 31] -ciò che tenderebbe a sottolinearne la sola dimensione statica- ma anche come suoi custodi attivi, che nella propria dimensione dinamica sono chiamati a svolgere una funzione creatrice di principi, valori e diritti costituzionali, una funzione di arbitrato nell'ambito dei conflitti che intervengano fra soggetti od organi costituzionali, in generale una funzione di rettifica del sistema complessivamente considerato finalizzata al perseguimento di due obiettivi fondamentali: colmare il fossato che talora esiste tra diritto e società e proteggere la democrazia [A. Barak, 2006, 231]. Tale funzione di riconduzione del diritto alla giustizia si sostanzia nella censura 
dell'operato del legislatore, è accompagnata dalla identificazione di principi, valori e diritti costituzionali che aggiorna e circostanzia le opzioni originariamente effettuate dal costituente, procedendo ove necessario alla necessaria ponderazione ed al conseguente bilanciamento degli interessi (sul versante della parte dogmatica, nonché su quello organizzativo ed ordinamentale delle Costituzioni) ed è eventualmente integrata dall'esercizio di ulteriori funzioni complessivamente finalizzate a garantire la perpetuazione della Costituzione nel tempo (risoluzione dei conflitti interorganici ed intersoggettivi, controllo sui partiti politici ecc.). La fonte di legittimazione di funzioni di tale entità qualitativa e quantitativa deve necessariamente essere ricercata al più alto livello e deve identificarsi con il potere costituente e con la Costituzione che il medesimo ha voluto. La giustizia costituzionale gode di legittimazione democratica in quanto contemplata in seno a Costituzione democratica venuta ad esistenza secondo un procedimento democratico. I costituenti che -soprattutto dopo il 1945- hanno accolto in seno alle Costituzioni contemporanee uno dei modelli di giustizia costituzionale ed in particolare il controllo di legittimità costituzionale delle leggi, hanno prospetticamente concepito gli organi di giustizia costituzionale quali propri commissari per il futuro, conferendo loro il mandato, sempre revocabile al verificarsi di evento qualificabile secondo la terminologia di Ackerman come momento costituzionale, a proteggere, adeguare e aggiornare le opzioni seguite in sede di adozione dell'atto fondativo dell'ordinamento. Gli organi di giustizia costituzionale partecipano più da vicino degli altri poteri costituiti della natura tipica del potere costituente.

I costituenti prendono atto, a partire dal secondo dopoguerra, della insufficienza del circuito rappresentativo tradizionale ad assicurare idonea rappresentazione e rappresentanza alla pluralità degli interessi emergenti in seno alla società e della sopravvenuta inadeguatezza della legge, divenuta -come già avevano avvertito Bachof e Forsthoff- sempre più contingente ed esposta alle umorali aspirazioni di minoranze occasionali, a selezionarli e ad assicurarvi appropriata tutela non arretrando rispetto ai tradizionali canoni della generalità ed astrattezza delle norme. Parlamenti divisi, pigri, temporeggiatori e incapaci di fornire idonea declinazione tecnica agli interessi rappresentati, nonché il progressivo atteggiarsi della legge quale legge-provvedimento sono fattori capaci di erodere fin dalle sue fondamenta lo Stato costituzionale democratico e di diritto.

La giustizia costituzionale nasce anche in conseguenza della profonda crisi di identità e funzionale che investe la legge ed i parlamenti nelle democrazie contemporanee e dalla volontà dei costituenti di preservare la democrazia e la giustizia al di là delle transeunti contingenze che tendono ad acquisire le forme di un diritto precario. 